\title{
Model identification and FE simulations: Effect of different yield loci and hardening laws in sheet forming
}

\author{
P. Flores ${ }^{\text {a }}$, L. Duchêne ${ }^{\text {b }}$, C. Bouffioux ${ }^{\text {e }}$, T. Lelotte ${ }^{\text {a }}$, C. Henrard ${ }^{\text {a }}$, N. Pernin ${ }^{\text {a }}$, A. Van Bad ${ }^{\text {c }}$, S. He $^{\text {c }}$, J. Duflou $^{\text {, }}$, \\ A.M. Habraken ${ }^{\mathrm{a}}$ \\ ${ }^{a}$ Department of ArGEnCO, University of Liège, Chemin des Chevreuils 1, B-4000 Liège, Belgium \\ ${ }^{b}$ Department of COBO, Royal Military Academy, Renaissancelaan 30, B-1000 Brussel, Belgium \\ ${ }^{c}$ Department of MTM, Katholieke Universiteit Leuven, Kasteelpark Arenberg 44, B-3001 Leuven, Belgium \\ ${ }^{d}$ Department of PMA, Katholieke Universiteit Leuven, Celestijnlaan 300B, B 3001 Leuven, Belgium \\ ${ }^{e}$ Department of MeMC - TW, Vrije Universiteit Brussel, Pleinlaan 2, 1050 Brussels, Belgium
}

\begin{abstract}
The bi-axial experimental equipment [Flores, P., Rondia, E., Habraken, A.M., 2005a. Development of an experimental equipment for the identification of constitutive laws (Special Issue). International Journal of Forming Processes] developed by Flores enables to perform Bauschinger shear tests and successive or simultaneous simple shear tests and plane strain tests. Flores investigates the material behavior with the help of classical tensile tests and the ones performed in his bi-axial machine in order to identify the yield locus and the hardening model. With tests performed on one steel grade, the methods applied to identify classical yield surfaces such as [Hill, R., 1948. A theory of the yielding and plastic flow of anisotropic materials. Proceedings of the Royal Society of London A 193, 281-297; Hosford, W.F., 1979. On yield loci of anisotropic cubic metals. In: Proceedings of the 7th North American Metalworking Conf. (NMRC), SME, Dearborn, MI, pp. 191-197] ones as well as isotropic Swift type hardening, kinematic Armstrong-Frederick or Teodosiu and Hu hardening models are explained. Comparison with the Taylor-Bishop-Hill yield locus is also provided. The effect of both yield locus and hardening model choices is presented for two applications: plane strain tensile test and Single Point Incremental Forming (SPIF).
\end{abstract}

Keywords: Anisotropic material; Constitutive behavior; Metallic material; Finite elements; Mechanical testing

\section{Introduction}

In practice, different metal forming processes such as deep drawing, stamping or bending are required to manufacture automotive parts, beverage or food cans, steel sheet panels used in aeronautics or civil engineering applications. Computer models try to replace the expensive and time-consuming trial-and-error methods used in conventional design. The Finite Element Method (FEM) is quite successful to simulate metal forming processes, but accuracy depends both on the constitutive laws used and their material parameters identification.

For instance, the final shape of a product is strongly linked to the plastic material flow and to the springback phenomenon. Plastic anisotropy explains the undulated rims called ears (Yoon et al., 2006), which appear in a cup produced by cylindrical tools applied on a circular blank. The classical isotropic von Mises yield criterion predicts no ears at all. The simple quadratic anisotropic Hill yield criterion often simulates an inaccurate fourearing profile. More complex models relying on the crystal plasticity and a homogenization approach provide results that are closer to the experimental observations, but they are quite greedy from a CPU time point of view.

This paper uses both simple classical phenomenological laws and micro-macro constitutive models based on crystal plasticity. Classical phenomenological models roughly consist in the fitting of functions on experimental results. They provide only crude tools, the quality of which depends both on the complexity of the chosen functions and on the type of experiments used to identify them.

The bi-axial experimental equipment (Flores et al., 2005a) developed by Flores enables to perform successive or simultaneous simple shear tests and plane strain tests (see Fig. 1). 
Such experiments applied on samples cut in different directions from the rolling direction investigate the onset of plasticity and identify the initial yield locus. Stress contours at identical plastic work can also be drawn. Such data coupled with the strain field measured by optical extensometer allows checking the associated flow rule. Stoughton and Yoon (2006) shows this usually accepted approach still need confirmation and they propose for aluminum and steel alloys accurate models of non-associated type. Next section shows the difference between experimental points and yield loci defined by a phenomenological model or deduced from texture measurement and crystal plasticity.

Kinematic hardening models are identified by cyclic shear tests showing the Bauschin-ger effect. Orthogonal tests are performed by successive simple shear and tensile tests. Such experimental results provide the necessary data to support simple models, like the one suggested by Armstrong and Frederick (1966) or more complex ones like the Teodosiu and Hu model (Teodosiu and Hu, 1995; Teodosiu and Hu, 1998; Bouvier et al., 2003; Haddadi et al., in press). Other deformation modes can be investigated for the identification of material parameters. (Brunet et al., 2001) used metal sheet bending-unbending tests and tensile tests for the identification of their model. (Hu, 2005; Tong, 2006) used standard uni-axial-tension and equal bi-axial-tension tests for the characterization of their models.

Fig. 1. Bi-axial machine designed at the ArGEnCO Laboratory, University of Liège.

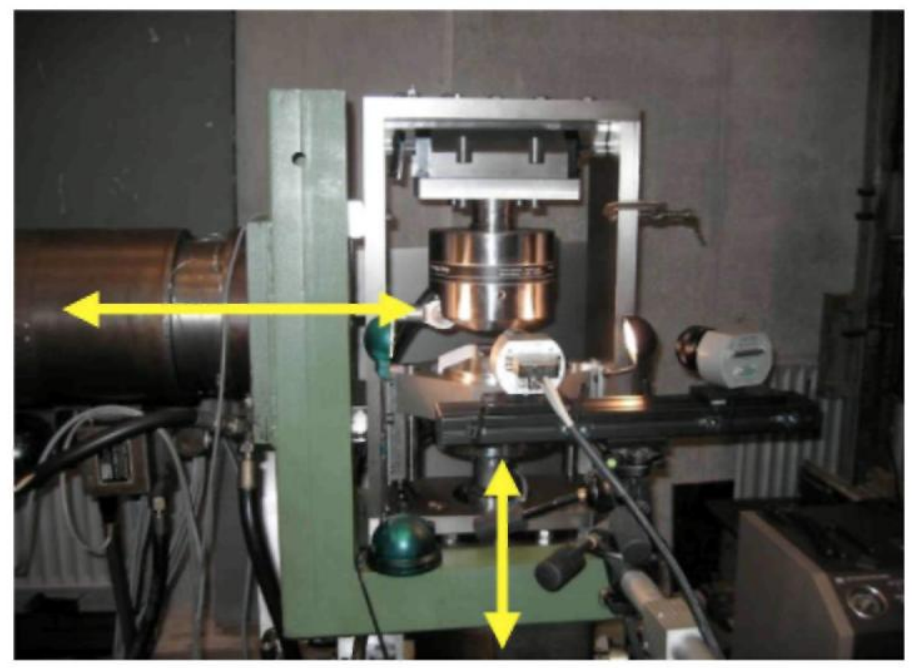

After the presentation of the method used to identify the yield locus and the hardening behavior for one material, two applications where the simulation results strongly depend on the model choice and identification are described: a plane strain tensile test and Single Point Incremental Forming (SPIF) process. The description of experimental procedure for all mechanical tests used in this article appears in Flores et al. (2005a) and Flores (2006).

\section{Model identification}

The first step is the identification of the initial yield locus shape and the second step is the hardening behavior. As underlined in the described identification approach, both steps cannot be completely decoupled. The case of DC06 IF steel sheet of $0.8 \mathrm{~mm}$ thickness is presented because this material shows a quite strong anisotropy. Tensile tests were performed in a standard tensile test machine of $20 \mathrm{kN}$ capacity (with a normalized specimen) while the plane strain and simple shear tests were performed in the bi-axial machine developed at the University of Liège (Flores et al., 2005b).

\subsection{Yield locus shape}

First, the texture was measured by X-ray diffraction and a set of 2000 representative crystal orientations was chosen (Van Houtte, 2002). The yield locus shape has been computed by a micro-macro approach: a full constraint Taylor model is applied where the crystal plasticity law is described by a Taylor-Bishop-Hill model (Van Houtte, 1988). Then, tensile, plane strain and simple shear tests were performed at $\alpha=0^{\circ}, 45^{\circ}$ and $90^{\circ}$ from the sheet Rolling Direction (RD) (see Fig. 2). The results of two different methods used to identify the Hill (1948) and the Hosford (1979) yield criteria are compared hereafter. The constant material parameters of these 
criteria must be defined according to an orthotropic material frame classically defined here as the Rolling Direction (RD, $X_{1}$-axis) and the Transversal Direction (TD, $X_{2}$-axis) of the sheet (see Fig. 2).

Fig. 2. Angle a with respect to the Rolling Direction, material axes $X_{1}, X_{2}, X_{3}$ or $R D, T D, N D$ and local axes $x_{1}$, $x_{2}, x_{3}$

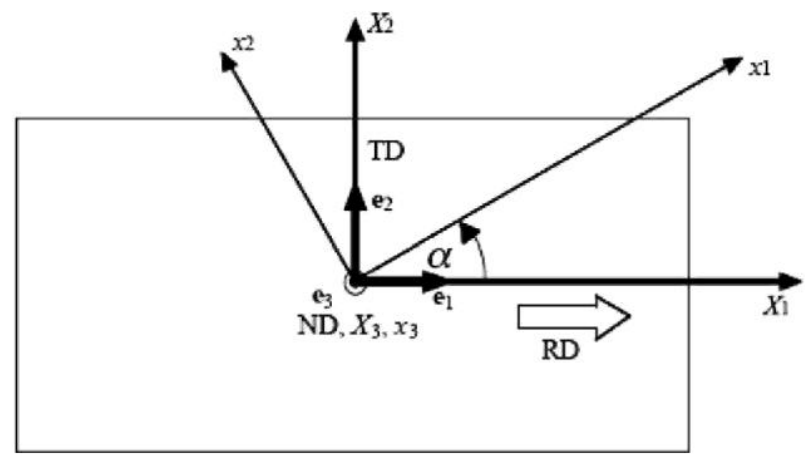

Eq. (1) defines the Hill (1948) yield criterion in plane stress state with the material parameters $H, G, F, N$ and the initial yield stress $R_{0}$ for a tensile test in the RD. The material parameters are defined for the criterion expressed in material axes $X_{1}, X_{2}, X_{3}$

$$
\mathscr{F}_{\text {Hill }}(\underline{\sigma})=\frac{1}{2}\left[(H+G) \sigma_{11}^{2}+(H+F) \sigma_{22}^{2}-2 H \sigma_{11} \sigma_{22}+2 N \sigma_{12}^{2}\right]-R_{0}^{2}=0
$$

Unlike the Hill (1948) yield criterion, the Hosford (1979) one is non-quadratic. Its main advantage is that exponent value fitting ensures a good approximation of experimental data (Banabic et al., 2000). Recommended values are $a=6$ for bcc materials and $a=8$ for fcc materials (Hosford, 1998). The main drawback of this criterion is the lack of shear stresses, as shown by Eq. (2) in plane stress

$$
\mathscr{F}_{\text {Hosford }}(\underline{\sigma})=H\left|\sigma_{11}-\sigma_{22}\right|^{a}+G\left|\sigma_{11}\right|^{a}+F\left|\sigma_{22}\right|^{a}-R_{0}^{a}=0
$$

The first identification method only uses the Lankford coefficients $r_{0}{ }^{\circ}, r_{45} \mathrm{O}, r_{90}{ }^{\circ}$ (i.e. the ratio between the transversal plastic strain rate and the thickness plastic strain rate) measured during tensile tests at $0^{\circ}, 45^{\circ}$ and $90^{\circ}$ from the RD. Note that accurate measurements of Lankford coefficients were done by Flores (2006). For this steel grade, the Lankford coefficients remain constant in the range of large plastic strains. Extensometers measure axial and transversal strains. The thickness strain is computed by volume conservation. Using associated plasticity theory, the flow rule defines the plastic strain rate proportional to the stress derivative of the yield locus, $\underline{\dot{c}}^{\mathrm{p}}=\dot{\lambda} \cdot \partial \mathscr{F} / \partial \underline{\sigma}$. Eqs. (3)-(5) can be established to identify the Hill material parameters, while the two first also provide the Hosford parameters:

$$
\begin{aligned}
& r_{0^{\circ}}=\frac{\dot{\varepsilon}_{2}^{\mathrm{p}}}{\dot{\varepsilon}_{3}^{\mathrm{p}}}=\frac{H}{G} \\
& r_{90^{\circ}}=\frac{\dot{\varepsilon}_{1}^{\mathrm{p}}}{\dot{\varepsilon}_{3}^{\mathrm{p}}}=\frac{H}{F} \\
& r_{45^{\circ}}=\frac{\dot{\varepsilon}_{22\left(\alpha=45^{\circ}\right)}^{\mathrm{p}}}{\dot{\varepsilon}_{3}^{\mathrm{p}}}=\frac{2 N-F-G}{2(F+G)}
\end{aligned}
$$

The relations $H+G=2$ for Hill (1948) and $H+G=1$ for Hosford (1979) complete the system of equations. The Lankford coefficients and the initial yield stress appear in Table 1 for the DC06.

The second identification method is mainly based on the plastic stresses measured by tensile, plane strain and simple shear tests. The plastic work $W_{\mathrm{p}}$ at $0.5 \%$ plastic strain is computed from the tensile test in RD. Contours of plastic work are defined from several yield stress states (tensile, plane strain and simple shear in the RD, TD and $45^{\circ}$ from the RD) computed at equal $W_{\mathrm{p}}$. These points are plotted in $\left(\sigma_{11}, \sigma_{22}, \sigma_{12}\right)$ axes and they belong to a surface defined by the chosen plastic work offset. For such small plastic strain levels, this surface approximates the yield locus. 
To define contours of plastic work, two hypotheses are assumed. Firstly, at small strains a simple shear test can represent a pure shear state. Secondly, the mechanical configuration of the bi-axial testing machine renders the measurement of the whole plane stress state impossible. The missing stress component is obtained using the flow rule and the total strain field measurements. The component $\sigma_{22}$, for a plane strain test performed in the $x_{1}$ direction (angle $\alpha$ from the RD), is related to the measured one $\sigma_{11}$ by:

$$
\dot{\varepsilon}_{22}^{\mathrm{p}}=\dot{\lambda} \frac{\partial \mathscr{F}}{\partial \sigma_{22}}=0
$$

This relation is expressed in the local axes defined in Fig. 2. The material parameters that define the shape of this surface are optimized in order to minimize the following function:

$$
\chi=\sum_{i=1, l}(1-\eta)\left[\frac{\sigma_{\text {Theo } \_}-\sigma_{\exp \__{-} i}}{R_{0}}\right]^{2}+\sum_{j=1, m} \eta\left[\frac{r_{\text {Theo }-j}-r_{\text {exp }-j}}{\bar{r}}\right]^{2}
$$

where the subscript $l$ defines the number of experimental tests used to measure stress points, and subscript $m$ the number of tests used to define anisotropy parameters. The subscripts Theo and exp refer respectively to the theoretical and the experimental values. $\eta$ is a weight factor for the stress and strain measurements. In what follows $\eta$ is set to 0 in order to focus the study on stress measurement. Finally, $\bar{r}$ is the average anisotropy coefficient

$\left(\bar{r}=\left(r_{0^{\circ}}+2 r_{45^{\circ}}+r_{90^{\circ}}\right) / 4\right)$

Tables 2 and 3 define for each criterion the experimental and numerical values compared to identify the material parameters.

Fig. 3 shows the Hill (1948) yield function fitted with Lankford coefficients (Hill-Lank-ford) or with the 8 experimental points (Hill-exp. points). Note that for this figure, the exact position of the plane strain points rely on relation (6) and the identified material parameters. These yield loci are compared with Taylor-Bishop-Hill (TBH) and (Hosford, 1979) surfaces. As underlined by Table 4 giving the parameters values, the (Hosford, 1979) yield loci identified by Lankford or stress point values are identical. The bi-axial stress state adimensional Kuwabara's points (Kuwabara et al., 1998), measured at different plastic strain levels $\left(\varepsilon^{p}=0.05 \%, 0.2 \%, 1 \%\right.$ and $4 \%$ ), coincide well with the Hosford and the Hill-exp. points surface predictions. One can observe that according to the experimental points, the quality of the Hill yield locus identified by Lankford values is quite poor. The Hill (1948) surface based on experimental stress points seems more accurate. The Hosford (1979) and TBH yield loci are close to each other.

Table 1. Lankford coefficients, yield stress for DCO6

\begin{tabular}{|c|l|l|l|}
\hline \multicolumn{1}{|c|}{$\boldsymbol{r}_{\mathbf{0}^{\circ}} \boldsymbol{r}_{\mathbf{9 0}}{ }^{\circ}$} & \multicolumn{1}{c|}{$\boldsymbol{r}_{\mathbf{4 5}^{\circ}}$} & $\left.\operatorname{Ro}_{\left(\mathbf{\varepsilon}_{\mathbf{0}}\right.}{ }^{\mathrm{p}}=\mathbf{0 . 5 \%}\right)[\mathrm{MPa}]$ \\
\hline 1.98 & 2.56 & 1.67 & 140.47 \\
\hline
\end{tabular}

\begin{tabular}{|c|c|c|}
\hline Test & Experimental point & Theoretical value \\
\hline Uni-axial tensile RD & $\sigma_{\text {exp_1 }}=\sigma_{11}(\alpha=0)$ & $\sigma_{11}=\sigma_{\text {Theo_1 }}=\sqrt{\frac{2}{H+G}} \sigma_{y}$ \\
\hline Uni-axial tensile TD & $\sigma_{\text {exp_2 }}=\sigma_{11}(\alpha=90)$ & $\sigma_{11}=\sigma_{\text {Theo_2 }}=\sqrt{\frac{2}{H+F}} \sigma_{\mathrm{y}}$ \\
\hline Uni-axial tensile $45^{\circ}$ & $\sigma_{\text {exp_3 }}=\sigma_{11}(\mathrm{a}=45)$ & $\sigma_{11}=\sigma_{\text {Theo_3 }}=\sqrt{\frac{8}{2 N+G+F}} \sigma_{\mathrm{y}}$ \\
\hline Plane strain $\mathrm{RD}^{\mathrm{a}}$ & $\sigma_{\text {exp_4 }}=\sigma_{11}(a .=0)$ & $\sigma_{11}=\sigma_{\text {Theo_4 }}=\sqrt{\frac{2}{H\left(1-k_{0}\right)^{2}+G+k_{0}^{2} F}} \sigma_{\mathrm{y}}$ \\
\hline Plane strain $\mathrm{TD}^{\mathrm{a}}$ & $\sigma_{\text {exp_5 }}=\sigma_{11}(\alpha=90)$ & $\sigma_{11}=\sigma_{\text {Theo_}} 5=\sqrt{\frac{2}{H\left(1-k_{90}\right)^{2}+k_{90}^{2} G+F}} \sigma_{\mathrm{y}}$ \\
\hline Plane strain $45^{\text {oa }}$ & $\sigma_{\text {exp_6}}=\sigma_{11}(\alpha=45)$ & $\sigma_{11}=\sigma_{\text {Theo_}} 6=\sqrt{\frac{8}{2 N\left(1-k_{45}\right)^{2}+\left(1+k_{45}\right)^{2}(G+F)} \sigma_{\mathrm{y}}}$ \\
\hline Simple shear RD & $\sigma_{\text {exp_7 }}=\sigma_{12}(\alpha=0)$ & $\sigma_{12}=\sigma_{\text {Theo_7 }}=\sqrt{\frac{1}{N} \sigma_{\mathrm{y}}}$ \\
\hline Simple shear $45^{\circ}$ & $\sigma_{\text {exp_8}}=\sigma_{12}(\alpha=45)$ & $\sigma_{12}=\sigma_{\text {Theo_ } 8}=\sqrt{\frac{2}{4 H+G+F}} \sigma_{\mathrm{y}}$ \\
\hline
\end{tabular}

Table 2. Experimental points and theoretical values for Hill (1948) yield criterion

${ }^{a}$ with $k_{0}=\frac{H}{H+F}, k_{90}=\frac{H}{H+G}$ and $k_{45}=\frac{2 N-G-F}{2 N+G+F}$. 
Table 3. Experimental points and theoretical values for Hosford (1979) yield criterion

\begin{tabular}{|l|l|l|}
\hline \multicolumn{1}{|c|}{ Test } & \multicolumn{1}{|c|}{ Experimental point } & \multicolumn{1}{|c|}{ Theoretical value } \\
\hline Uni-axial tensile RD & $\sigma_{\text {exp_1 }}=\sigma_{11}(\alpha=0)$ & $\sigma_{11}=\sigma_{\text {Theo_1 }}=\left(\frac{1}{H+G}\right)^{1 / a} \sigma_{\mathrm{y}}$ \\
\hline Uni-axial tensile TD & $\sigma_{\text {exp_2 }}=\sigma_{11}(\alpha=90)$ & $\sigma_{11}=\sigma_{\text {Theo_2 }}=\left(\frac{1}{H+F}\right)^{1 / a} \sigma_{\mathrm{y}}$ \\
\hline Plane strain RD & $\sigma_{\text {exp_3 }}=\sigma_{11}(\mathrm{a}=0)$ & $\sigma_{11}=\sigma_{\text {Theo_3 }}=\left(\frac{1}{H\left|1-k_{0}\right|^{a}+G+\left|k_{0}\right|^{a} F}\right)^{1 / a} \sigma_{\mathrm{y}}$ \\
\hline Plane strain TD & $\sigma_{\text {exp_4 }}=\sigma_{11}(\alpha=90)$ & $\sigma_{11}=\sigma_{\text {Theo_4 }}=\left(\frac{1}{H\left|k_{90}-1\right|^{a}+\left|k_{90}\right|^{a} G+F}\right)^{1 / a} \sigma_{\mathrm{y}}$ \\
\hline Simple shear 45 & $\sigma_{\text {exp_5 }}=\sigma_{12}(\alpha=45)$ & $\sigma_{12}=\sigma_{\text {Theo_5 }}=\left(\frac{1}{2^{a} H+G+F}\right)^{1 / a} \sigma_{\mathrm{y}}$ \\
\hline
\end{tabular}

${ }^{a}$ With $k_{0}=1 /\left(1+(F / H)^{1 /(a-1)}\right.$ and $k_{90}=1 /\left(1+(G / H)^{(1 / a-1))}\right.$.

Fig. 3. Scaled yield loci in principal stress directions in material axes $X_{1}, X_{2}$ and experimental points.

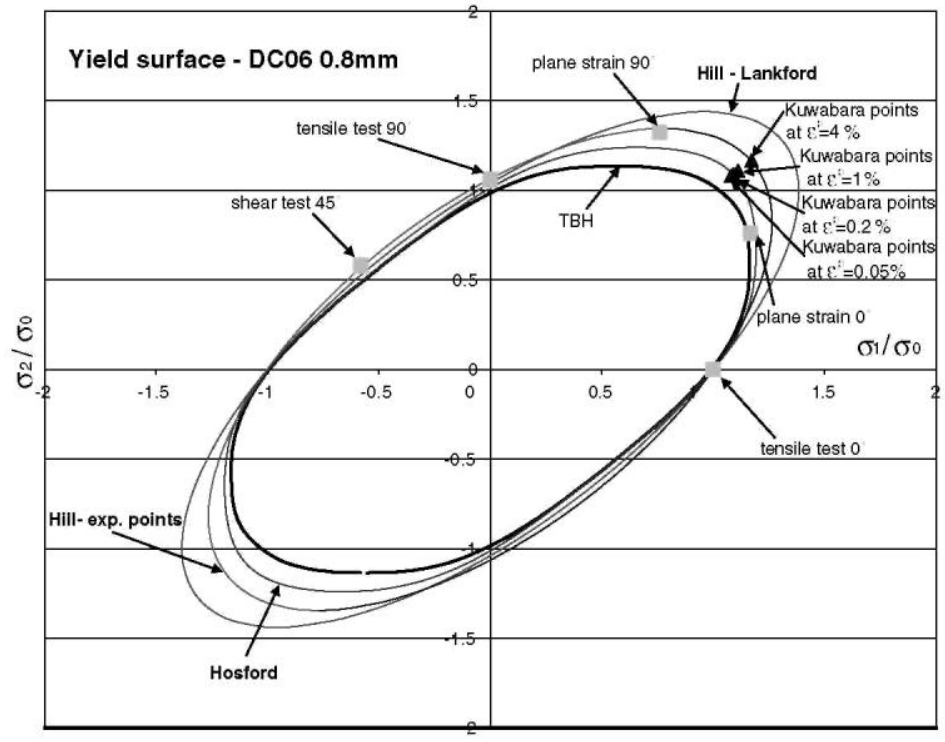

Table 4. Material parameters for DC06 steel

\begin{tabular}{|l|l|l|l|l|l|l|}
\hline \multicolumn{1}{|c|}{ Yield function } & \multicolumn{1}{c|}{ Identification method } & \multicolumn{1}{c|}{$\boldsymbol{F}$} & \multicolumn{1}{c|}{$\boldsymbol{G}$} & \multicolumn{1}{c|}{$\boldsymbol{N}$} & $\boldsymbol{a}$ \\
\hline Hill (1948) & Lankford coefficients & 1.33 & 0.52 & 0.67 & 2.58 & - \\
\hline Hill (1948) & Experimental points & 1.13 & 0.69 & 0.87 & 2.76 & - \\
\hline Hosford (1979) & Lankford coefficients & 0.66 & 0.26 & 0.34 & - & 6 \\
\hline Hosford (1979) & Experimental points & 0.66 & 0.25 & 0.34 & - & 6 \\
\hline
\end{tabular}

All the experimental points are plotted in Fig. 4, which shows sections of TBH and Hill exp. points yield loci at shear stress level 0, 57 and $72 \mathrm{MPa}$. The pure shear case is $82.4 \mathrm{MPa}$ in the test, when predicted values by TBH and Hill are respectively 76.75 and $83 \mathrm{MPa}$.

These results show that mechanical tests reproducing other stress states than pure tension are required to obtain more accurate material parameters identification. 


\subsection{Hardening model}

\subsubsection{Introduction}

In order to reach a good agreement of the model for tensile tests, monotonic shear tests and reverse shear tests, three hardening models are identified: a pure kinematic hardening of Armstrong-Frederick (AF) type (Armstrong and Frederick, 1966) and two mixed isotropic-kinematic hardening models (isotropic Swift approach coupled with AF and Teod-osiu-Hu model).

Fig. 4. Sections in Hill and TBH yield loci at shear stress level 0, 57 and 72 MPa compared with corresponding experimental points in material axes $X_{1}, X_{2}, X_{3}$.

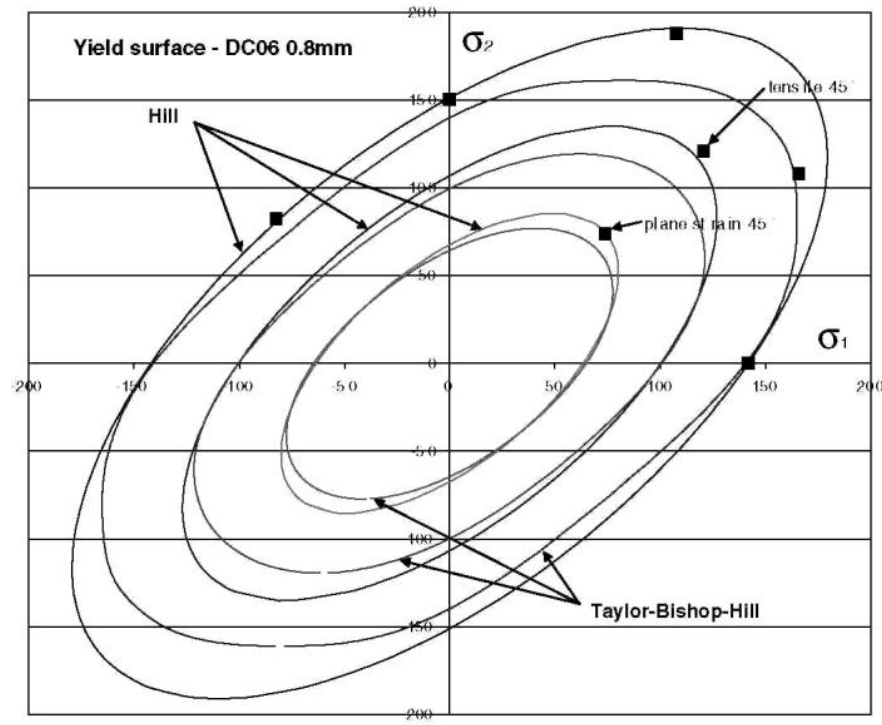

The Hill (1948) parameter $N$ identified in previous section (line 2 of Table 4) was able to accurately predict the experimental point of initial yielding in pure shear state. However for the three studied hardening models, the only way to recover experimental stress-strain curves for both monotonic tensile test and simple shear test in RD was to modify this $N$ parameter from 2.76 to 3.26 value. The other parameters $H, F, G$ are kept constant, which means that the section in principal stress directions (Fig. 3) is not modified and remains close to experimental points. This $N$ parameter defining the pure shear state is quite sensitive. For instance, its value deduced from the Taylor-Bishop-Hill yield locus drawn in Fig. 4 and associated to initial texture is 3.35.

Assuming that texture is the main factor responsible for the initial yield locus shape seems right. Indeed, Fig. 4 shows a quite good agreement between the TBH yield locus shape associated with the initial texture and experimental points. In the applied procedure to identify hardening parameters, the yield locus shape and by consequence the texture are assumed constant. Is this hypothesis reliable or not?

The fact that during uni-axial tensile tests with DC06 steel, experimental Lankford coefficients remained constant, confirms that only a weak texture evolution took place. However is this conclusion true during a simple shear test? Probably not, as non-negligible texture evolutions during simple shear tests were reported by Peeters et al. (2001) and Lelotte et al. (2005) for other steel grades.

During a simple shear test, studied for instance by Bouvier et al. (2005), the shear component $\sigma_{12}$ is not alone, both $\sigma_{11}$ and $\sigma_{22}$ components are non-negligible (see Fig. 5). The stress and strain fields as well as the texture evolution have been computed by one finite element simulation using the micro-macro law MINTY (Habraken and Duchêne, 2004).

The MINTY model computes only a small zone of the yield locus, which is updated when its position is no longer located in the part of interest of the yield locus or when the yield locus changes due to texture evolution.

In this constitutive micro-macro law, the plastic criterion and the stress integration scheme are not defined by a yield locus formulation. It uses a linear stress-strain interpolation in the five-dimensional (5D) deviatoric stress space described by Eq. (8): 


$$
\underline{s}=\tau \underline{L} \underline{u}
$$

where $\underline{s}$ and $\underline{u}$ are 5D vectors containing respectively the deviatoric part of the stress (the hydrostatic part being elastically computed according to Hooke's law) and the deviatoric plastic strain rate direction (unit vector). $\tau$ is a scalar describing the work hardening according to:

$$
\tau=K\left(\Gamma^{0}+\Gamma\right)^{n}
$$

where $K, \Gamma^{0}$, and $n$ are material parameters identified by experimental data and $\Gamma$ is the cumulated polycrystal induced slip.

Fig. 5. Stress state during a simple shear test in RD.

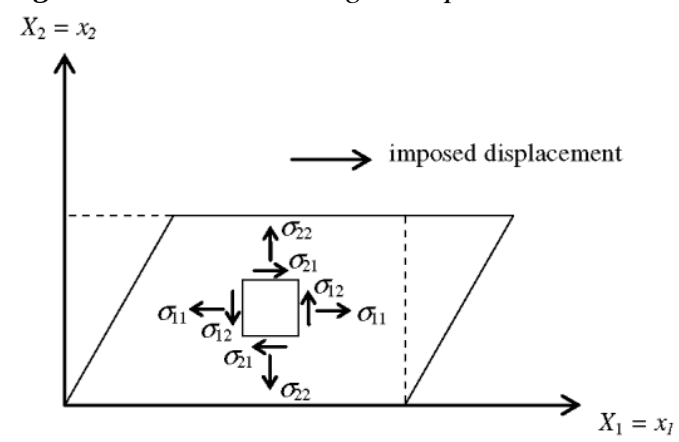

The macroscopic anisotropic interpolation is included in matrix $\underline{L}$. Its identification relies on 5 directions of $\underline{u}^{*}$ advisedly chosen in the deviatoric strain rate space and their associated deviatoric stresses $\underline{s}^{*}$ computed by a polycrystal plasticity model. This micro-macro model uses Taylor's assumption of equal macroscopic strain and microscopic crystal strain (full constraints Taylor's model). It computes the average of the response of a set of representative crystals evaluated with a microscopic model, taking into account the plasticity at the slip systems level. The stress vectors $\underline{s}^{*}$ lie on the yield locus. They define the vertices of the interpolation domain and are called stress nodes. The matrix $\underline{L}$ is built on the basis of these 5 stress nodes. More details as well as validation and applications of MINTY model can be found in Habraken and Duchêne (2004).

The FE simulation of monotonic simple shear test of DC06 using MINTY model confirms yield locus shape evolution. Initial, intermediate and final textures are extracted and used to compute the pure shear stress state evolution with Taylor-Bishop-Hill model. The Hill (1948) $N$ parameter is then fitted on these stresses. The results show that its initial value 3.35 continuously decreases to 3.20 . These computations confirm that the value 3.26 identified from the experimental curves measured in tensile and simple shear tests can be used as the Hill (1948) model assumes constant yield locus shape and requires a mean $N$ value. So, Table 5 reminds the Hill parameters used in further identification procedure.

\subsubsection{Pure kinematic hardening law of Armstrong-Frederick}

First, a simple kinematic hardening model of the Armstrong and Frederick (1966) type is used to model the shift of the yield locus without any size evolution.

A second-order tensor $\underline{X}$, known also as back-stress, describes the displacement of the yield surface during plastic deformation. The yield locus size, which remains constant, is given by $R_{0}$ in Eq. (10) and represents the initial yield stress defined in a tensile test in RD direction:

$$
\mathscr{F}\left(\underline{\sigma}, \underline{X}, R_{0}\right)=\bar{\sigma}\left(\underline{\sigma}^{\prime}-\underline{X}\right)-R_{0}=0
$$

where $\underline{\sigma}$ is the deviatoric stress tensor. The evolution of the back-stress $\underline{X}$ is defined by Eq. 


$$
\underline{\nabla}=C_{X} \cdot\left(X_{\mathrm{SAT}} \cdot \underline{\dot{\varepsilon}}^{\mathrm{p}}-\dot{\bar{\varepsilon}}^{\mathrm{p}} \cdot \underline{X}\right)
$$

where $C_{x}$ is the parameter describing the rate of kinematic hardening, $X_{\mathrm{SAT}}$ the saturation value and $\dot{\bar{\varepsilon}}^{\mathrm{p}}$ is the anisotropic equivalent plastic strain rate.

Table 5. Hill (1948) material parameters for DC06 steel

\begin{tabular}{|c|c|c|c|c|}
\hline $\boldsymbol{H}$ & \multicolumn{1}{|c|}{$\boldsymbol{G}$} & $\boldsymbol{G}$ & \multicolumn{1}{c|}{$\boldsymbol{N}$} \\
\hline 1.13 & 0.69 & 0.87 & 3.26 \\
\hline
\end{tabular}

The material parameters are fine-tuned up using an optimization method that is mainly based on Marquardt's algorithm (Marquardt, 1963) in order to fit the stress-strain experimental curves obtained from tensile, simple shear tests and the Bauschinger shear experiment with $10 \%$ pre-strain. The model predictions are computed by 'one finite element' simulation with the home-made FEM package Lagamine developed by the ULg team (Habraken and Cescotto, 1990). As the number of material parameters is limited, no specific strategy is required to identify the parameters. The set of parameters is given in Table 6 .

\subsubsection{Mixed hardening law (Swift + Armstrong-Frederick)}

Secondly, a mixed hardening model is used. This type of hardening describes both the size and displacement evolution of the yield surface. The constitutive equations are composed of Eqs. (12) and (13)

$$
\mathscr{F}(\underline{\sigma}, \underline{X}, k)=\bar{\sigma}\left(\underline{\sigma}^{\prime}-\underline{X}\right)-k\left(\bar{\varepsilon}^{\mathrm{p}}\right)=0
$$

The material parameters are identified using the monotonic tensile test at the RD, the monotonic simple shear test at the RD and the Bauschinger test at the RD with a $10 \%$ amount of pre-strain.

$$
k\left(\bar{\varepsilon}^{\mathrm{p}}\right)=K\left(\varepsilon_{0}+m \cdot \bar{\varepsilon}^{\mathrm{p}}\right)^{n}
$$

The evolution of the back-stress $\underline{X}$ is defined by Eq. (11). As the number of material parameters increases, some care to keep physical sense of the parameters is required during the use of the optimization approach to identify the parameters. The set of parameters is given in Table 7 .

\subsubsection{Mixed Teodosiu and Hu hardening law}

Thirdly, a more complex mixed (isotropic-kinematic) hardening model of Teodosiu type is used (Haddadi et al., in press). This phenomenological model is based on microscopic and macroscopic experimental observations. It is capable of describing monotonic large strains as well as the influence of strain-path changes and the amount of pre-strain on the flow stress. This model uses a set of four internal variables $(\underline{X}, \underline{P}, \underline{S}, R)$, and 13 material parameters are required. The back-stress $\underline{X}$ is a second-order tensor having the dimension of the stress. The polarity $\underline{P}$ is a non-dimensional second-order tensor. A fourth-order tensor $\underline{\underline{S}}$ describes the directional strength of planar persistent dislocation structures. The choice of its order is due to the need to describe the anisotropic contribution of persistent dislocation structures to the flow stress. Finally, a scalar internal variable $R$, with the dimension of the stress, takes into account the isotropic hardening caused by the statistically distributed dislocations.

Table 6. Kinematic Armstrong-Frederick material parameters for DC06

\begin{tabular}{|c|l|l|}
\hline \multicolumn{1}{|c|}{$\boldsymbol{R}_{\boldsymbol{0}}$ (MPa) } & \multicolumn{1}{c|}{$\boldsymbol{C}_{\boldsymbol{x}}$} & \multicolumn{1}{c|}{$\mathbf{X}_{\mathrm{SAT}}(\mathbf{M P a})$} \\
\hline 140.47 & 12.29 & 115.20 \\
\hline
\end{tabular}

Table 7. Kinematic mixed material parameters for DCO6

\begin{tabular}{|l|l|l|l|l|l|l|}
\hline $\boldsymbol{R}_{\boldsymbol{0}}(\mathbf{M P a})$ & $\boldsymbol{K}(\mathbf{M P a})$ & $\boldsymbol{\varepsilon}_{\mathbf{0}}$ & $\boldsymbol{n}$ & $\boldsymbol{m}$ & $\boldsymbol{C}_{\boldsymbol{x}}$ & $\mathbf{X}_{\mathrm{SAT}}(\mathbf{M P a})$ \\
\hline 140.47 & 499.9 & 0.0063 & 0.25 & 0.715 & 12.7 & 16.75 \\
\hline
\end{tabular}


The yield surface is described by the following equation:

$$
\mathscr{F}(\underline{\sigma}, \underline{X}, \underline{P}, \underline{\underline{S}}, R)=\bar{\sigma}\left(\underline{\sigma}^{\prime}-\underline{X}\right)-R_{0}-R-m|\underline{\underline{S}}|=0
$$

where $R_{0}$ is the initial yield stress and $m|\underline{\underline{S}}|$ is the contribution of the persistent dislocation structures to the isotropic hardening, with $m \in[0,1]$. The back-stress is ruled by the following equation:

$$
\stackrel{\nabla}{X}=C_{X}\left[\frac{X_{\mathrm{SAT}}}{\bar{\sigma}}\left(\underline{\sigma}^{\prime}-\underline{X}\right)-\underline{X}\right] \dot{\bar{\varepsilon}}^{\mathrm{p}}
$$

The dependence of $\underline{X}$ on the persistent dislocation structures is included in the scalar function $X_{\mathrm{SAT}}(\underline{\underline{S}}, \underline{N})$ :

$$
X_{\mathrm{SAT}}=X_{\mathrm{SAT} 0}+(1-m)|\underline{\underline{S}}| \sqrt{q+(1-q) \beta_{S}^{2}}
$$

with $\beta_{S}=S_{\mathrm{D}} / \underline{\underline{S}} \mid, S_{\mathrm{D}}=\underline{N}: \underline{\underline{S}}: \underline{N}$ and $\underline{N}=\dot{\bar{\varepsilon}}^{\mathrm{p}} /\left|\dot{\bar{\varepsilon}}^{\mathrm{p}}\right|$

The evolution of $S_{\mathrm{D}}$ follows the equation:

$$
\dot{S}_{\mathrm{D}}=C_{\mathrm{SS}}\left[g\left(S_{\mathrm{SAT}}-S_{\mathrm{D}}\right)-h S_{\mathrm{D}}\right] \dot{\bar{\varepsilon}}^{\mathrm{p}}
$$

Functions $g(\underline{P}, \underline{N})$ and $h(\underline{X}, \underline{N})$ reduce the evolution of $S_{\mathrm{D}}$ upon stress reversal

$$
h=\frac{1}{2}\left(1-\frac{\underline{X}: \underline{N}}{X_{\mathrm{SAT}} \frac{\underline{\sigma^{\prime}-\underline{X}}}{\bar{\sigma}}: \underline{N}}\right)
$$

and

$$
g= \begin{cases}1-\frac{C_{p}}{C_{\mathrm{SD}}+C_{p}}\left|\frac{S_{\mathrm{D}}}{S_{\mathrm{SAT}}}-P_{\mathrm{D}}\right| & \text { if } P_{\mathrm{D}} \geqslant 0 \\ \left(1+P_{\mathrm{D}}\right)^{n_{p}}\left(1-\frac{C_{p}}{C_{\mathrm{SD}}+C_{p}} \frac{S_{\mathrm{D}}}{S_{\mathrm{SAT}}}\right) & \text { otherwise. }\end{cases}
$$

The tensor $\underline{\underline{S}}_{\mathrm{L}}$, associated with the latent part of the persistent dislocation structures evolves in the following way:

$$
\stackrel{\nabla}{\underline{S_{\mathrm{L}}}}=-C_{\mathrm{SL}}\left(\frac{Z}{S_{\mathrm{SAT}}}\right)^{n_{\mathrm{L}}} \underline{\underline{S}}_{\underline{\mathrm{L}}} \dot{\bar{\varepsilon}}^{\mathrm{p}}
$$

with

$$
Z=\left|\underline{\underline{S_{L}}}\right|=\sqrt{|\underline{\underline{S}}|^{2}-S_{\mathrm{D}}^{2}}
$$

The evolution law for the polarity tensor is:

$$
\stackrel{\nabla}{P}=C_{p}(\underline{N}-\underline{P}) \dot{\bar{\varepsilon}}^{\mathrm{p}}
$$

The set of 13 material parameters present in this model is given in Table 8 . The number of material parameters is now important and some parameters are clearly dedicated only to some events (stagnation of hardening in reverse test, in orthogonal tests,...). Details of the developed identification procedure can be found in Flores (2006).

Figs. 6-9 compare the model predictions using the different sets of material parameters with experimental results. For the tensile test (Fig. 6), the best fit is obtained by the mixed Teodosiu hardening. The pure kinematic hardening model of the Armstrong-Frederick type is definitely worse. In the case of simple shear test (Fig. 6), 
the best prediction is also given by the mixed Teodosiu hardening. This one is closer to experimental values than the mixed (Swift $+\mathrm{AF}$ ) hardening. Once again, the model of AF type is worse. Note that gamma is defined as the tangent of the shear angle.

Fig. 7 presents Bauschinger shear test after $10 \%$ of pre-shear using the three different hardening models. One can notice that pure AF hardening model gives bad results, it is not appropriate at all. Results are better using either a mixed hardening or a Teodosiu one.

Consider now Fig. 8 which shows four Bauschinger tests at different amounts of pre-strain. These tests highlight the presence of the Bauschinger effect, as well as the influence of the amount of pre-strain on the flow stress stagnation when reversing the load.

Table 8. Teodosiu and Hu hardening parameters for DC06

\begin{tabular}{|c|c|c|c|c|c|c|c|c|c|c|c|c|}
\hline $\begin{array}{c}\boldsymbol{R}_{\mathbf{0}} \\
(\mathbf{M P a})\end{array}$ & $\boldsymbol{C}_{\boldsymbol{R}}$ & $\boldsymbol{R}_{\mathrm{SAT}}(\mathbf{M P a})$ & $\boldsymbol{C}_{\boldsymbol{x}}$ & $\boldsymbol{X}_{\mathrm{SATO}}(\mathbf{M P a})$ & $\boldsymbol{m}$ & $\boldsymbol{q}$ & $\boldsymbol{C}_{\mathrm{SD}}$ & $\boldsymbol{C}_{\mathrm{SL}}$ & $\boldsymbol{S}_{\mathrm{SAT}}(\mathbf{M P a})$ & $\boldsymbol{n}_{\mathrm{p}}$ & $\boldsymbol{n}_{\mathrm{L}}$ & $c_{\mathrm{p}}$ \\
\hline 140.47 & 19.2 & 67.7 & 150 & 21.56 & 0.3 & 2.69 & 3.27 & 4.61 & 246.83 & 50 & 0.38 & 1.67 \\
\hline
\end{tabular}

Fig. 6. Tensile test and shear test simulated by mixed hardening model (Swift + AF), pure kinematic ArmstrongFrederick hardening, mixed Teodosiu hardening and experimental curves.

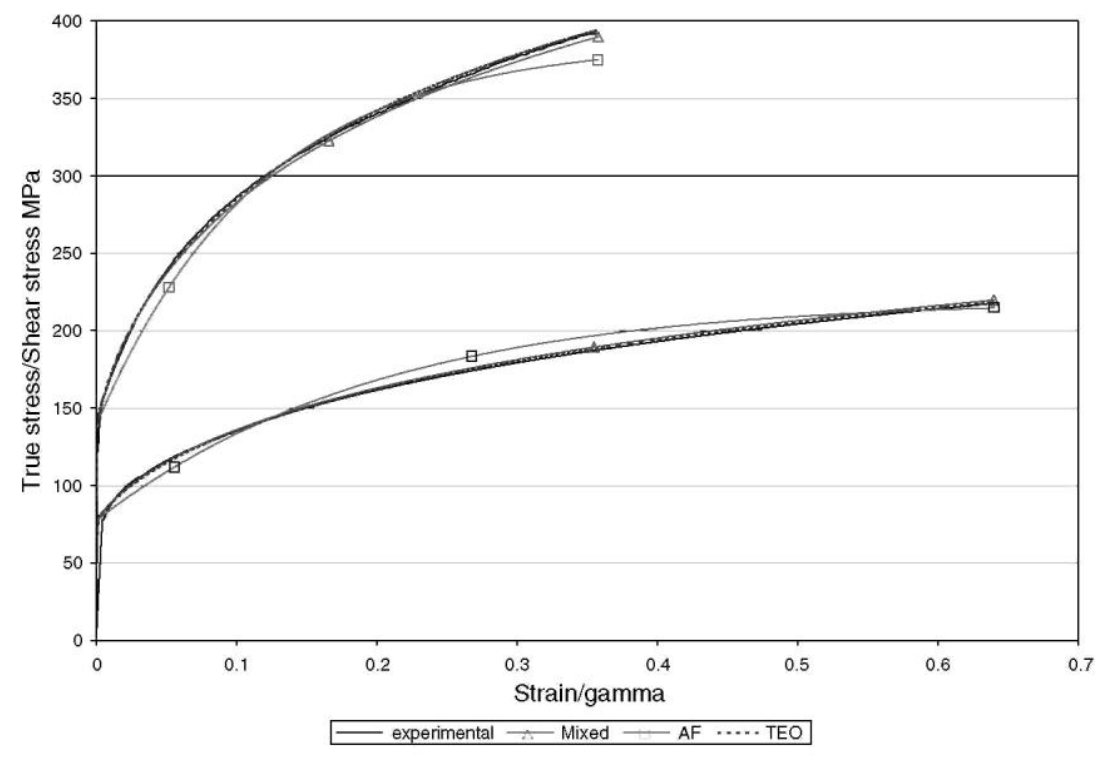

Fig. 7. Bauschinger shear test after $10 \%$ of pre-shear and simulation predictions by a mixed, a kinematic AF and a Teodosiu hardening.

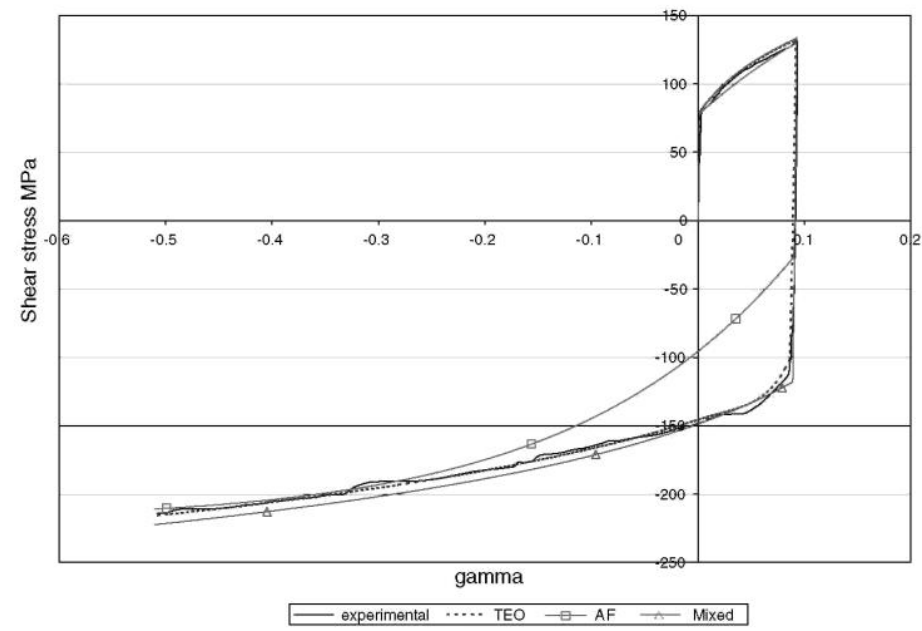


Fig. 8. Bauschinger tests at different amount of pre-strain.

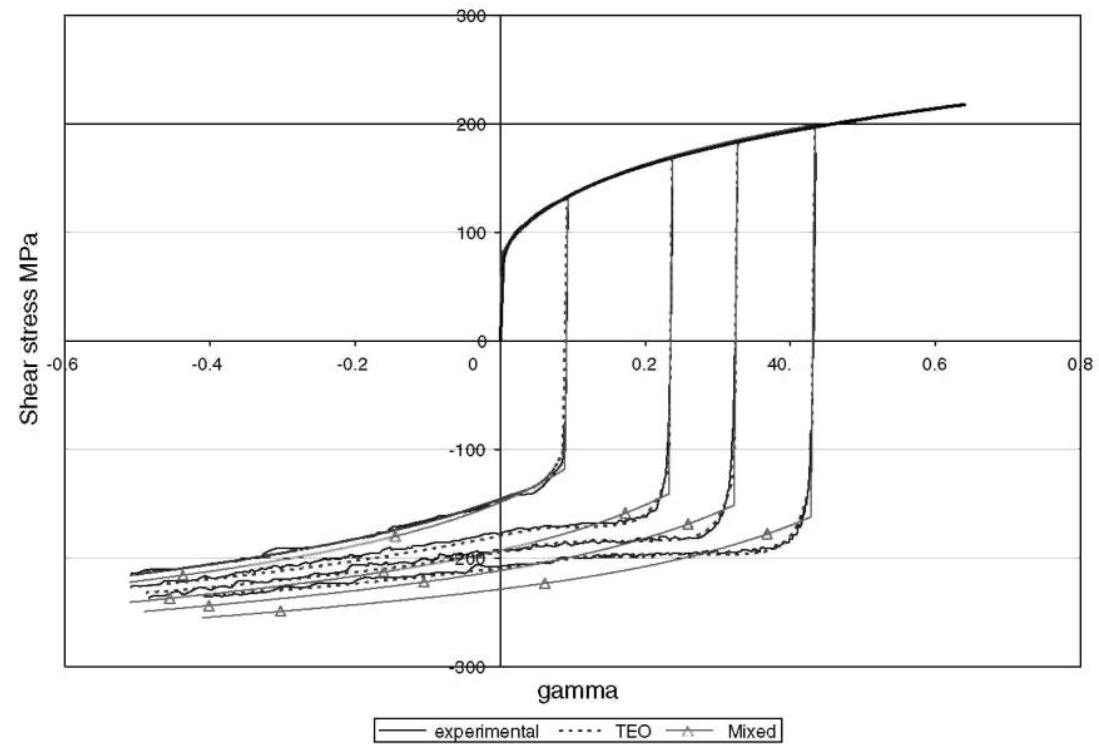

Fig. 9. Orthogonal test, plane strain test followed by a simple shear test.

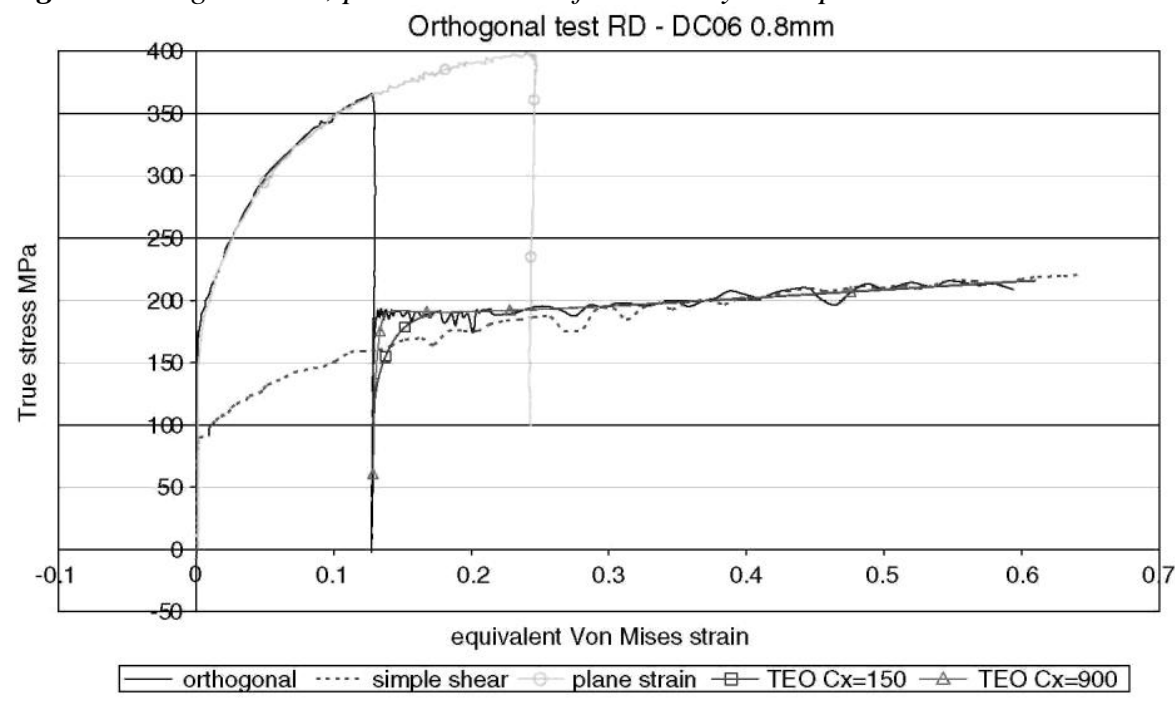

Firstly, one can notice that the mixed hardening law does not allow correct predictions of the work-hardening stagnation. Secondly, the Teodosiu hardening law is in good agreement with experimental results.

Note that only the Teodosiu and Hu hardening model is able to predict the so-called cross effect (increase of the yield stress after an orthogonal strain-path change) present in an orthogonal test. Fig. 9 shows such a test: a plane strain test interrupted and followed by a simple shear test. Experimental monotonic tests are plotted as well as the orthogonal test and the prediction by Teodosiu's model for the second loading curve. Note that the material parameter $C_{x}$ defines both the curvature for the second loading in a Bauschinger shear test and the orthogonal test. The value of 150 is perfect for Bauschinger test when a value of 900 is better for the orthogonal test. So clearly Teodosiu's model could be improved to solve this problem.

Fig. 10 provides a last comparison. It shows the hardening model of Teodosiu coupled with three different hardening: Hill yield locus of Table 5 (identified by stress experimental point and with $N$ parameter adjusted), Hill yield locus of line 2 of Table 4 (identified by stress experimental point and with $N$ parameter not adjusted), and of first line of Table 4 (identified by Lankford coefficient). The constitutive model identified using Lankford coefficients predicts a higher shear stress than the approach using experimental data. The results predicted by the model identified by stress values with $N$ parameter adjusted coincide well with the experimental curve. 
Fig. 10. Tensile test and shear test simulated by mixed Teodosiu with Hill (1948) identified by Lankford coefficient, simulated by mixed Teodosiu with Hill (1948) identified by experimental data and experimental curves.

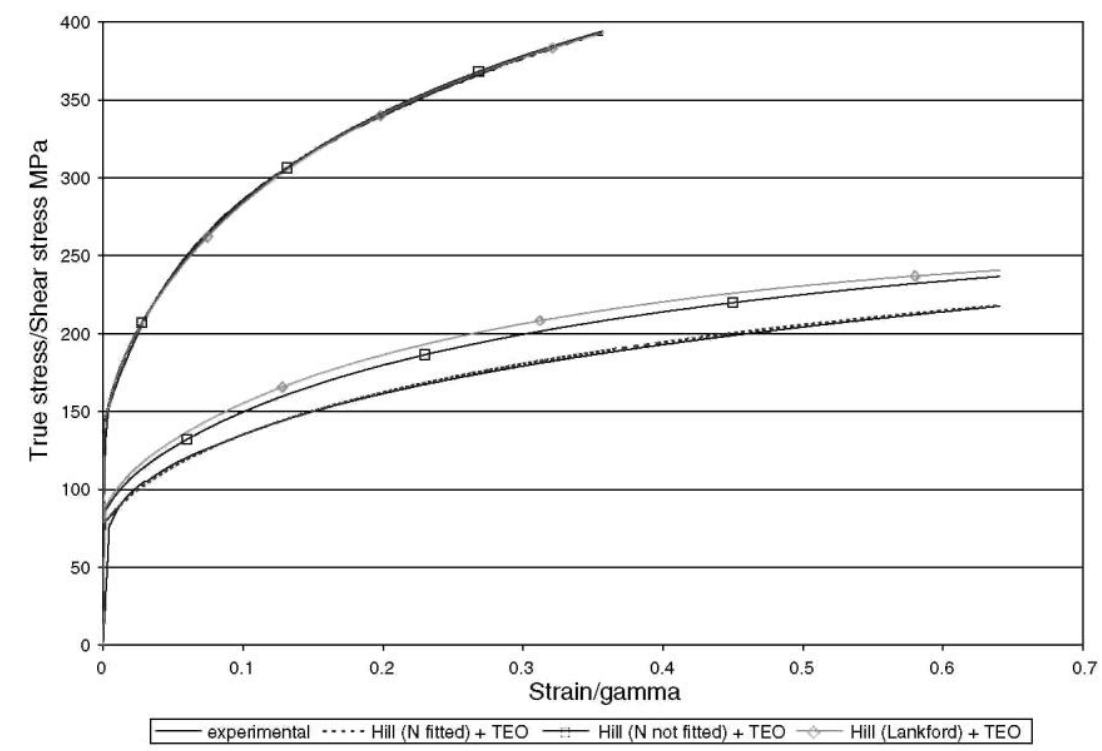

\section{Applications}

\subsection{Plane strain test, validation}

\subsubsection{Introduction}

The model identified in previous section is used to simulate a plane strain test performed on the DC06 material. The experimental measures allow checking which model is closer to the actual material behavior.

Fig. 11 presents the specimen geometry designed for the bi-axial machine. Dimensions of the initial specimen are $100 \times 30 \mathrm{~mm}$. The dotted area $(30 \times 3 \mathrm{~mm})$ is the measurable zone. The chosen geometry allows performing successively plane strain test and simple shear test (see Fig. 9). The deformation is imposed by the pistons (vertical or horizontal) of the bi-axial testing machine. Here for the studied plane strain test in the RD direction, only a vertical displacement is imposed.

The strain field can be measured in the whole deformation zone (by the optical strain gauge), and the average stress is computed from the load cell data and the actual area. This last assumption is only valid if the stress field is homogeneous.

The stress tensor is represented by:

$$
\underline{\sigma}=\sigma_{11} \underline{e}_{1} \otimes \underline{e}_{1}+\sigma_{22} \underline{e}_{2} \otimes \underline{e}_{2}
$$

with

$$
\begin{aligned}
\sigma_{11}=\frac{F_{\text {vertical }}}{A_{\text {actual }}} \rightarrow \text { measured by vertical load cell } \\
\rightarrow \text { optical measurements }+ \text { volume conservation }
\end{aligned}
$$

$F_{\text {Vertical }}$ is the load measured by the load cell and $A_{\text {actual }}$ is the actual area of the specimen deduced from optical measurement and volume conservation at the central line. The stress measured in that way will be called "global" stress and represents the average stress over the deformation zone. The component $\sigma_{22}$ cannot be measured with this mechanical configuration. 
Fig. 11. Specimen geometry and deformation area.

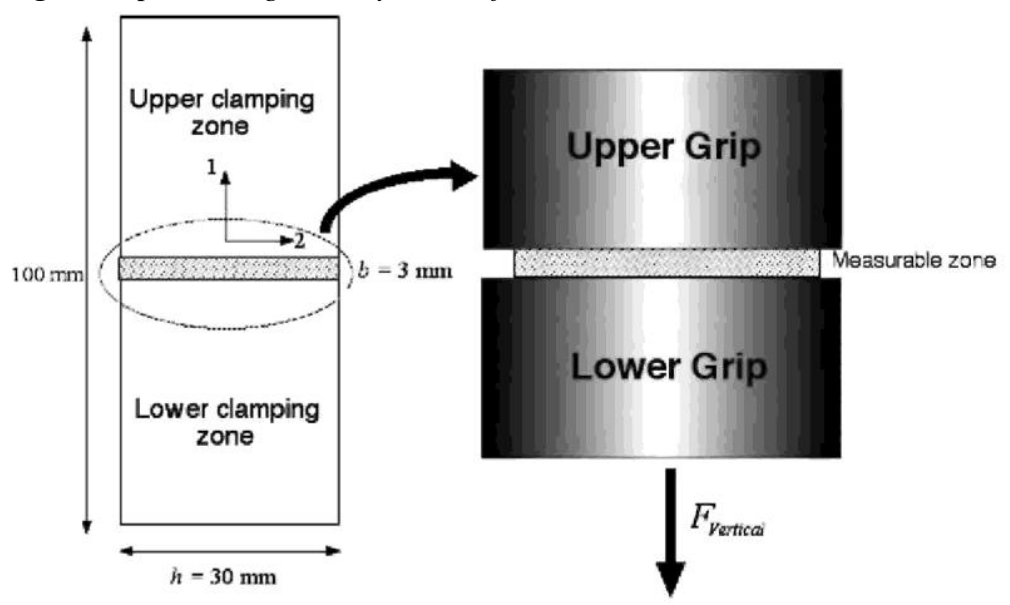

\subsubsection{Experimental results}

Fig. 12 shows the experimental strain field distribution at the centerline level of the sample for the respective logarithmic strain components $\varepsilon^{\text {In }}{ }_{11}$ and $\varepsilon_{22}^{\text {In }}$ at different strains.

One can notice that the strain tensor is homogeneous on the deformation area except at the free edges where horns appear. The homogeneity depends on the amount of strain $\varepsilon_{11}^{\text {In }}$. As the strain component $\varepsilon_{22}^{\text {In }}$ is negligible in the homogeneous zone of the deformation area, the expected plane strain state is actually reached. These results are confirmed by Figs. 13 and 14 which present measured strain fields in the plane strain test respectively for $\varepsilon_{11}=16 \%$ and $\varepsilon_{11}=20 \%$.

Note that horns were expected according to the explanation of Fig. 15 but seem quite high (Fig. 12).

It can be explained by the initiation of a crack near the grips between $\varepsilon_{11}=10 \%$ and $\varepsilon_{11}=15 \%$. At level $\varepsilon_{11}=$ $20 \%$, the crack is quite large and justifies the size of the horns, see Fig. 16. Such crack comes from ripping at the grip level. Note that a notched specimen could prevent such failure but it is not used here as samples are designed for both shear and plane strain tests.

\subsubsection{Simulations results}

In order to validate the parameters established in Tables 5 and 8, a finite element simulation of the plane strain test from previous section is performed with the home-made FEM package Lagamine (Habraken and Cescotto, 1990). Table 9 summarizes the finite element simulation data. The chosen element for this simulation is an eightnode mixed-type brick element with one integration point called BWD3D (Duchêne et al., 2005) using the new approach to prevent volume and shear locking as well as deformation without energy (hourglass modes) developed by Wang and Wagoner (2004).

Another important aspect in the FE code Lagamine is the method used to determine the position of the local reference axes. These local axes are a convenient way to fulfill the objectivity of the derivatives of state variables tensors and stress tensor. They allow modeling the anisotropic material behavior during the large rotations involved in FE simulations.

The way to determine this local reference system is based on the Constant symmetric local velocity gradient method developed by Munhoven and Habraken (1995). This method concerns the computation of the velocity gradient. Usually its symmetric part is the well-known strain rate tensor and its skew-symmetric part is the spin tensor. According to the Constant symmetric local velocity gradient method, the velocity gradient is assumed to be constant during each FE time step. This assumption imposes the strain path. The constant value of the velocity gradient can be determined from the configurations at the beginning of the step and the assumed velocity field. 
If it is expressed in the global reference system (which always remains fixed during the FE simulation), the so obtained constant velocity gradient is generally non-symmetric. The symmetry of the velocity gradient can however be obtained by expressing it in another reference system. In other words, in this method the symmetry condition fixes the position of the local reference system. As a consequence, the spin tensor corresponding to the symmetric constant velocity gradient is equal to zero while it is expressed in this local reference system. Conveniently, the integration of the constitutive law in these local axes is incrementally objective without the necessity of Jaumann type corrections. Furthermore, the local reference system is also assumed to correspond to the material axes where material properties are defined.

Fig. 12. $\varepsilon_{11}^{I n}$ and $\varepsilon_{22}^{\text {In }}$ components at the centerline for different levels of major strains in plane strain test (DC06 $0.8 \mathrm{~mm}$ thick).

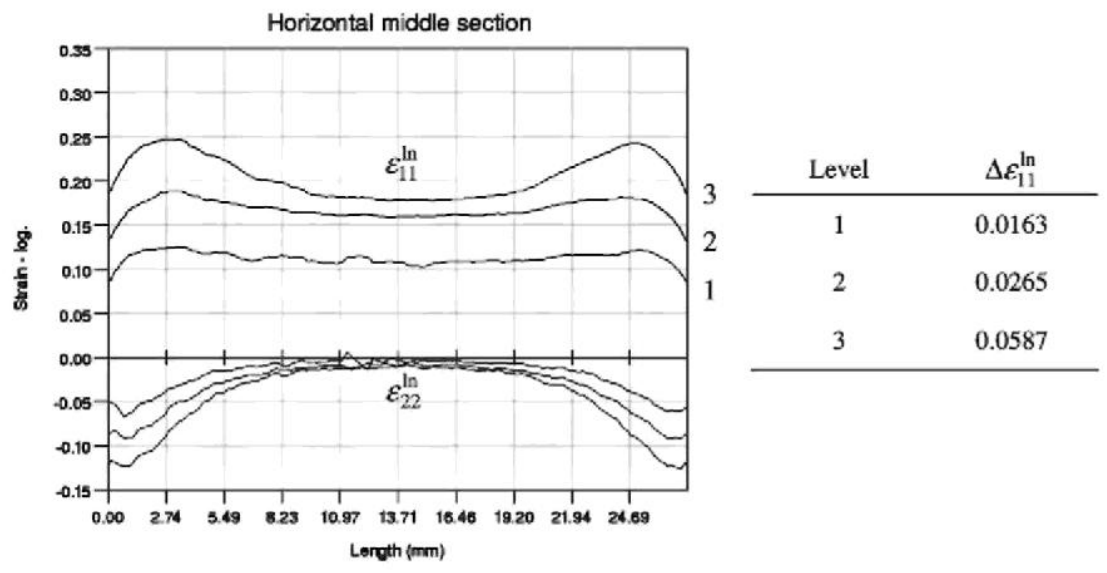

Fig. 13. Major strain field for a plane strain test for a $\varepsilon_{11}=16 \% .0 C 060.8 \mathrm{~mm}$.

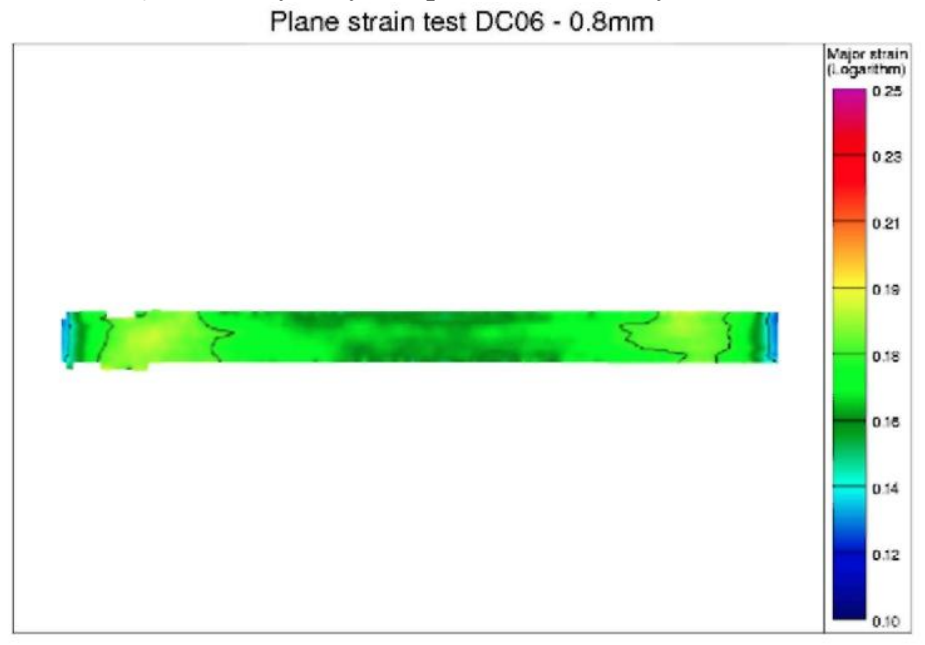

Fig. 14. Major strain field for a plane strain test for a $\varepsilon_{11}=20 \% .0 C 060.8 \mathrm{~mm}$. Plane strain test DCO6 $-0.8 \mathrm{~mm}$

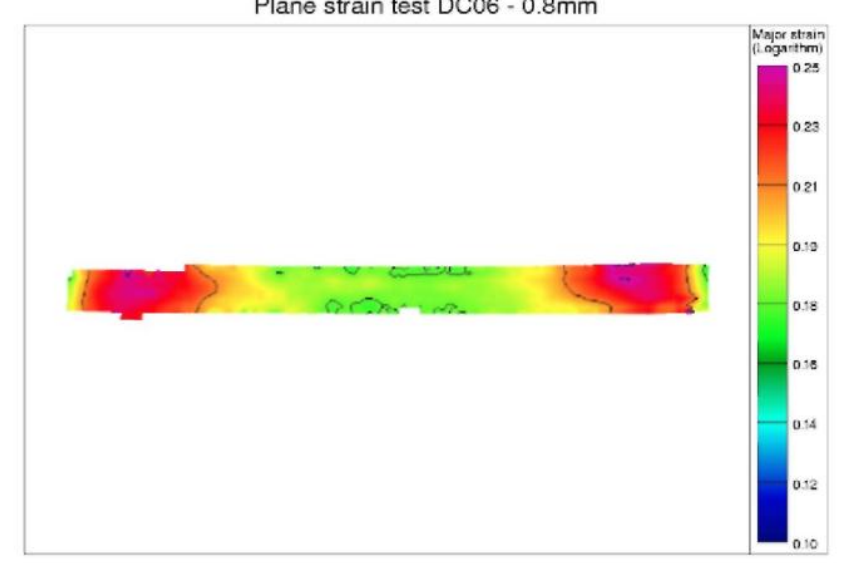


Fig. 15. Evolution of $\varepsilon_{11}$ strain distribution with dimensions of the sample shape.

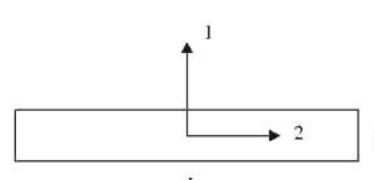

$h$

sample shape

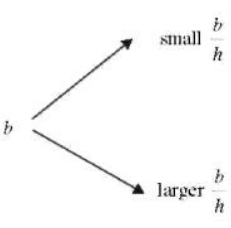

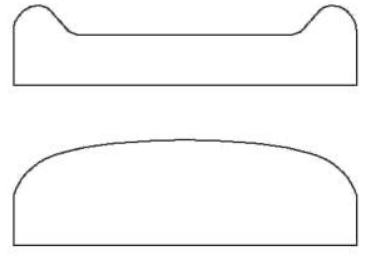

$\varepsilon_{11}$ strain distribution

Fig. 16. Deformed specimen for a plane strain test for a $\varepsilon_{11}=20 \%$. DC06 $0.8 \mathrm{~mm}$.

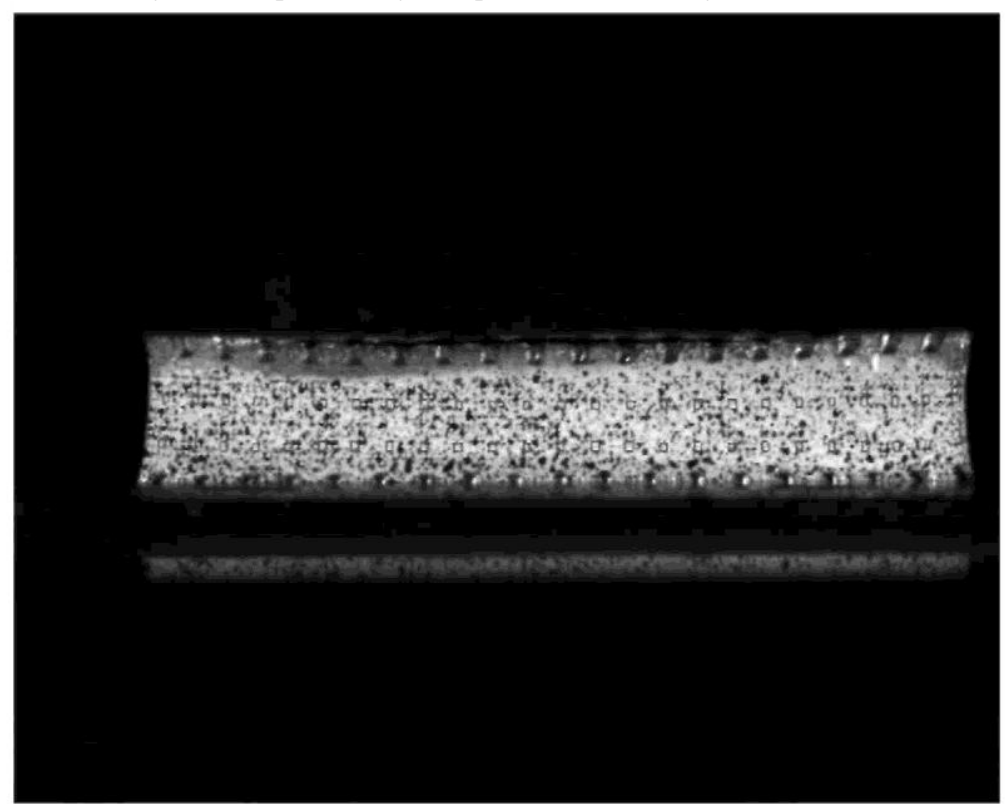

Table 9. Finite element strategy

\section{Finite element simulation data}

\begin{tabular}{|l|l|}
\hline Code & Lagamine developed since 1984 at ArGEnCO Department \\
\hline Element type & BWD3D-1 integration point \\
\hline Constitutive law & $\begin{array}{l}\text { Hill3D_KI (Hill (1948) + Teodosiu and Hu hardening law) and } \\
\text { MINTY law coupled with Teodosiu }\end{array}$ \\
\hline Material & DC06 $0.8 \mathrm{~mm}$ thick \\
\hline
\end{tabular}

In order to model any heterogeneity in the test, a refined mesh of 707 elements is used.

The experimental data are then compared with the results of stress-strain curves computed with:

- the Hill (1948) surface identified by Lankford coefficients (Table 4 and Fig. 3): Hill Lankford;

- the Hill (1948) surface identified by experimental stress points at $\varepsilon^{\mathrm{P}}{ }_{0}=0.5 \%$ (Tables 4 and 5): Hill-exp. points;

- the MINTY law (with initial texture and no texture evolution during the simulation) (see Fig. 3).

Each yield locus is coupled with the Teodosiu and Hu hardening law. These three models predict similar strain fields. All results confirm the horn strain distribution. Fig. 17(a) validates the horn level with measured strain field while some discrepancy appears already in Fig. 17(b).

In order to be consistent with the experimental test, the stress is computed from the resulting force divided by the actual area given in the FE simulation.

Fig. 18 compares the results. It can be seen that the constitutive model Hill-Lankford predicts a higher stress than 
the other approaches. The results predicted by Hill-exp. points and MINTY are closer to experimental curve up to a strain of $10 \%$. Beyond this amount of strain, the crack apearing in the test justifies that curves diverge.

Fig. 17. Strain field for plane strain test (a) for a $\varepsilon_{11}=10 \%$, (b) for a $\varepsilon_{11}=16 \%$. DC06 $0.8 \mathrm{~mm}$.

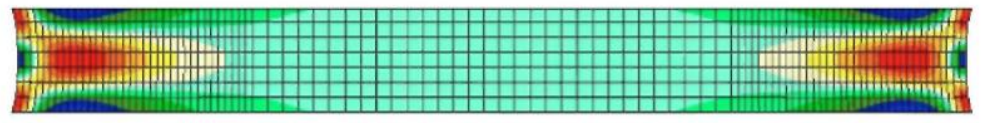

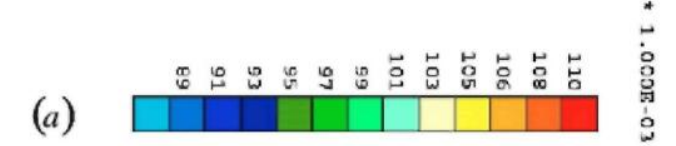
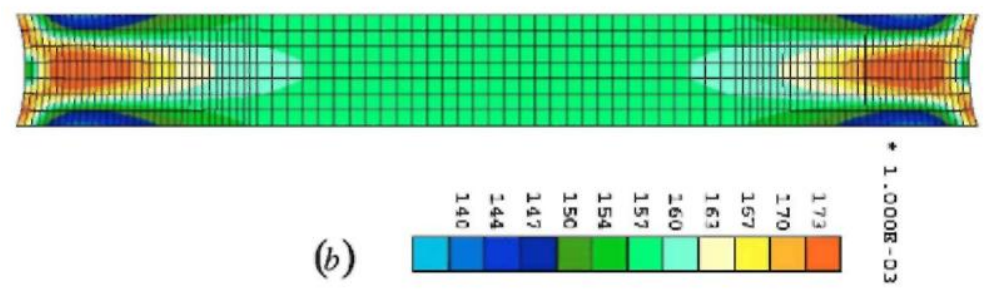

Fig. 18. Plane strain test. DC06 $0.8 \mathrm{~mm}$.

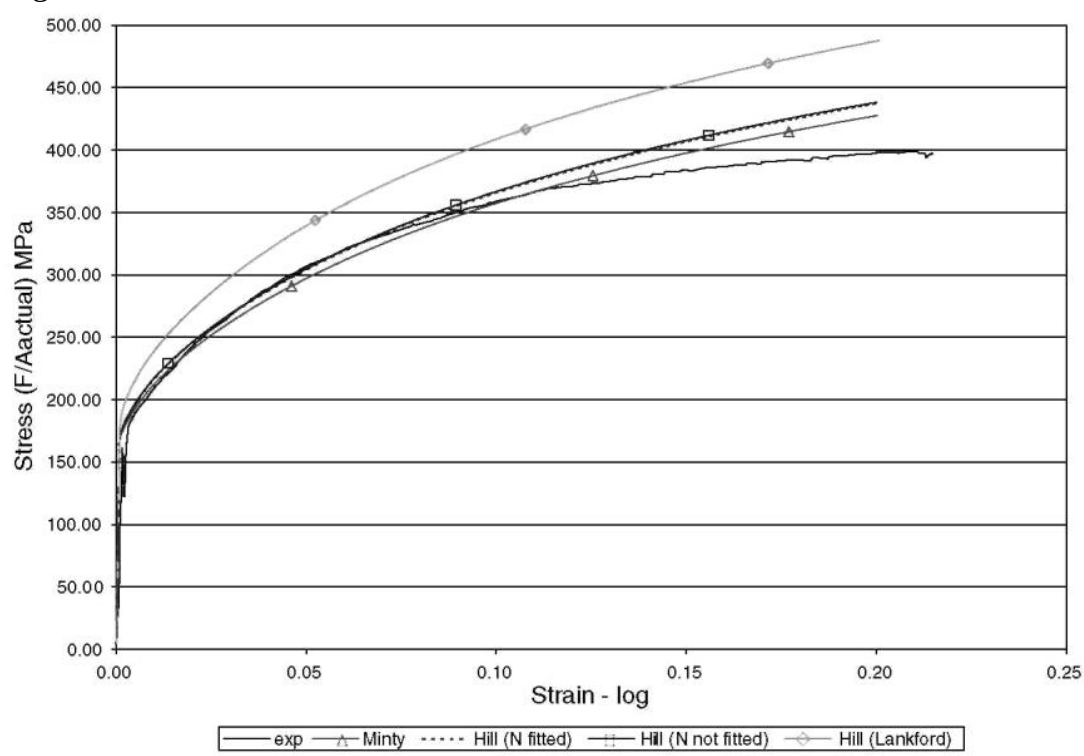

\subsection{Single point incremental forming}

\subsubsection{Process description}

The incremental sheet forming process has emerged in the past few years as a potential alternative to conventional sheet metal stamping processes. The process uses a smooth-ended tool under numerical control to create a local indentation in a clamped sheet. By dragging the point of contact around the sheet according to a programmed tool path, a wide variety of shapes may be formed without the need for specific tooling. Two versions of the process have been explored: with and without a supporting post on the reverse side of the workpiece. This section will only focus on the latter approach, called "single point" incremental forming (SPIF) presented in Fig. 19.

The review paper (Jeswiet et al., 2005) summarizes some fundamental research work carried out to investigate the deformation mechanism and material behavior. FEM simulations of this process are quite heavy as the tool path is very long and, if no remeshing module with numerous refinement and coarsening steps is used, a refined mesh is necessary everywhere as the tool induces very localized plastic strain. 


\subsubsection{Case study}

Fig. 20 presents the Single Point Incremental Forming process of a cone of aluminum alloy AA 3003-O. The dimensions of the initial sheet are $225 \times 225 \times 1.2 \mathrm{~mm}$. The cone wall angle is $50^{\circ}$ and the cone depth $40 \mathrm{~mm}$. The steel tool has a diameter of $12.7 \mathrm{~mm}$ and its vertical step size is $0.5 \mathrm{~mm}$. Tool forces were measured during the process (Duflou et al., 2005) and the final shape was measured offline by laser scanning.

Fig. 19. Single point incremental forming process.
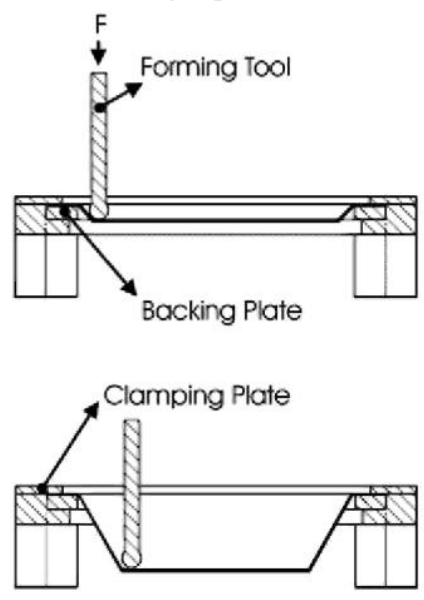

Fig. 20. SPIF process of one $50^{\circ}$ cone.
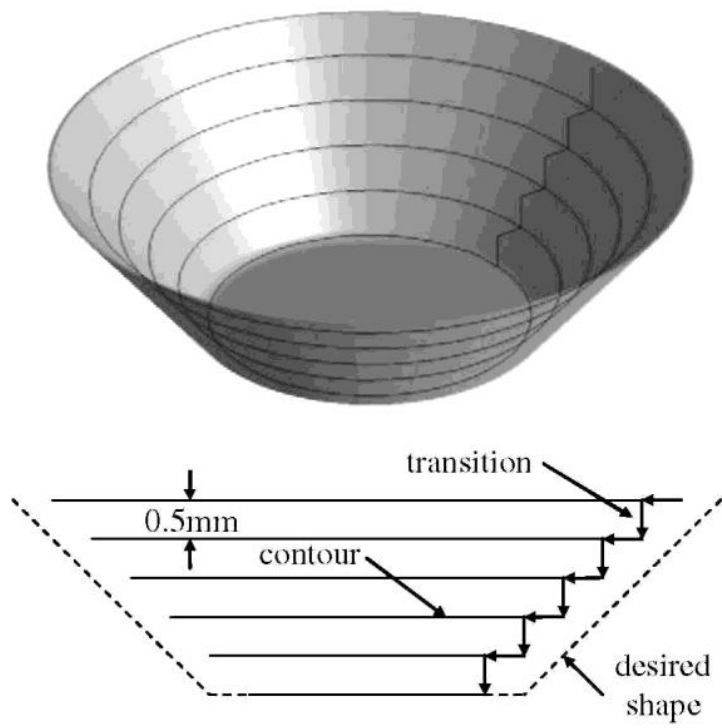

The flow stress curve of the material obtained by tensile tests is approximated by the Swift law $\sigma=180(\varepsilon+$ $0.00109)^{0.21}(\mathrm{MPa})$. Two different yield criteria were used: (Hill, 1948) identified from the Lankford coefficients $r_{0}=0.68, r_{90}=0.66$ and $r_{45}=0.73$, and the isotropic von Mises law.

\subsubsection{Geometrical simulations results}

All the SPIF simulations commented hereafter are performed with the commercial FEM package Abaqus/Standard by the K.U.Leuven team.

In Abaqus, the integration of stress-rate is done in the co-rotational material axes (Li et al., 2003).

Brick elements with reduced number of integration points (element type C3D6 and C3D8R in the terminology of Abaqus) are utilized for the blank. The mesh consists of three layers of elements through the thickness. It covers either a $40^{\circ}$ pie shape as presented in Fig. 21 , a $90^{\circ}$ pie or the full circular blank. The simulations reported in this section only use the above isotropic Swift hardening model, effect of hardening model is analyzed in next section. 
The results from Henrard et al. (2005), He et al. (2005a) and He et al. (2005b), confirmed by other research teams (Fratini et al., 2004; Iseki, 2001), show that concerning the exact shape prediction at the end of the process, the type of constitutive models is not a key factor. The discrepancy between the prediction from a $40^{\circ}$ pie mesh and the experiment is shown in Fig. 22. FEM simulation is accurate in the wall region but predicts a cone bottom that is too deep compared to experimental results.

However, for the same conical shape, it has been demonstrated that using a $90^{\circ}$ pie mesh or a full circular blank improves the prediction. The actual process is metric and symmetry boundary conditions induce additional stiffness that spoils the accuracy, see Fig. 23 (He et al., 2005b).

Fig. 21. Mesh used to simulate Cone SPIF process.

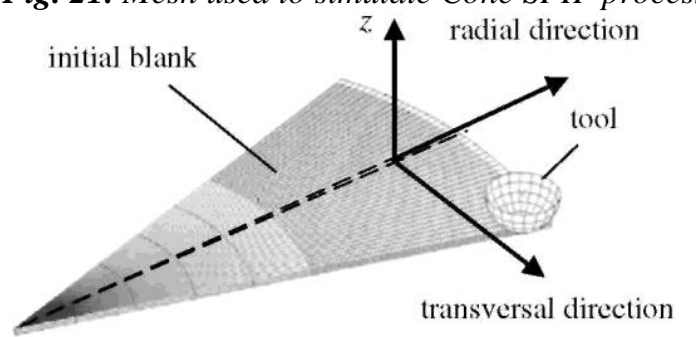

Fig. 22. Top surface of the cross section of the formed cone.

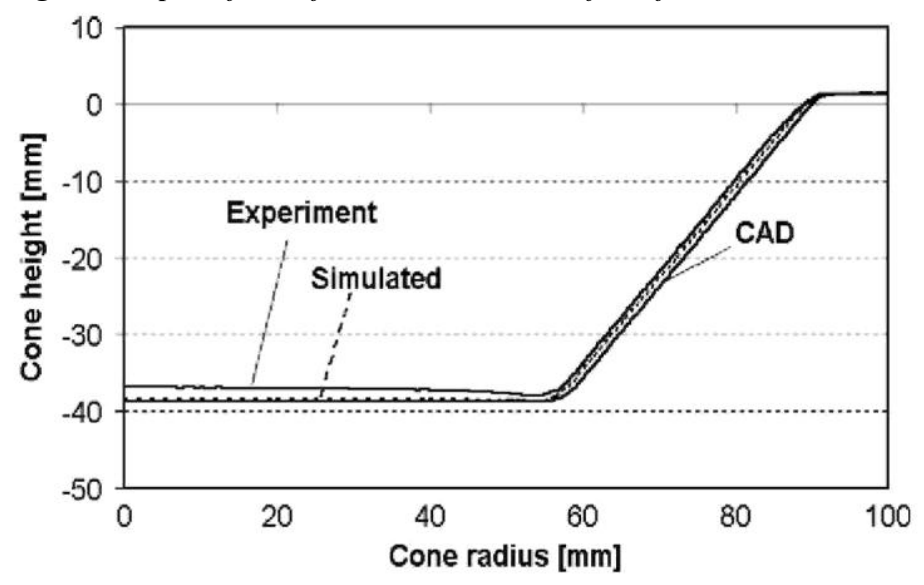

Fig. 23. Comparison of resulting geometries obtained with partial and full model simulations using Abaqus (at an intermediate step; upper and lower sheet layers are plotted).

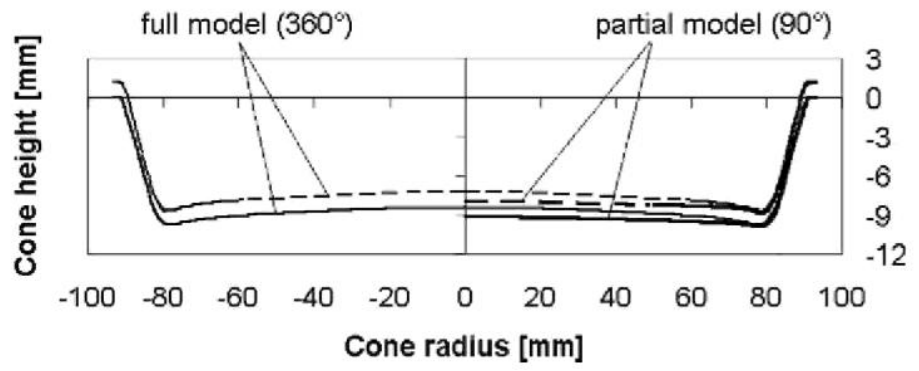

In comparison with the effect of the model geometry, the effect of the constitutive model is smaller, as shown in Fig. 24.

\subsubsection{Force analysis}

Tool force measurements during the process allow another validation of the FEM simulations (He et al., 2005a). When the mesh is sufficiently refined, the force level is not dependent on the dimensions of the piece. Models of $40^{\circ}, 90^{\circ}$ pie or full $360^{\circ}$ circular blank using Von Mises or Hill constitutive laws with isotropic hardening have provided nearly identical results: a prediction about $30 \%$ higher than the measurements.

To understand this difference, the strain history of one material point has been analyzed during the process in a 
local coordinate system that co-rotates with the finite element. In Fig. 25, the strain history of one element localized at the origin of the local tangential axis defined in Fig. 21 has been drawn. The oscillations result from the fact that during each circular tool path 'arc' at one vertical level, strain only appears when the tool is near or on this element. It is clear that the absolute values of the axial (thickness direction), radial and tangential components increase stepwise during the process as shown in Fig. 25. However, it is noticed that the tangential strain is very close to zero through the process, which confirms the plane strain assumption for the SPIF process.

Knowing this strain history, the material behavior has been analyzed in more details. First the yield locus shape computed by the TBH model has been drawn and compared to Hill, Von Mises and Hosford models in Fig. 26. Here axis 1 is the rolling direction and 2 the transversal direction in the sheet plane.

Fig. 24. Comparison of resulting geometries with Von Mises or Hill (1948) constitutive laws.

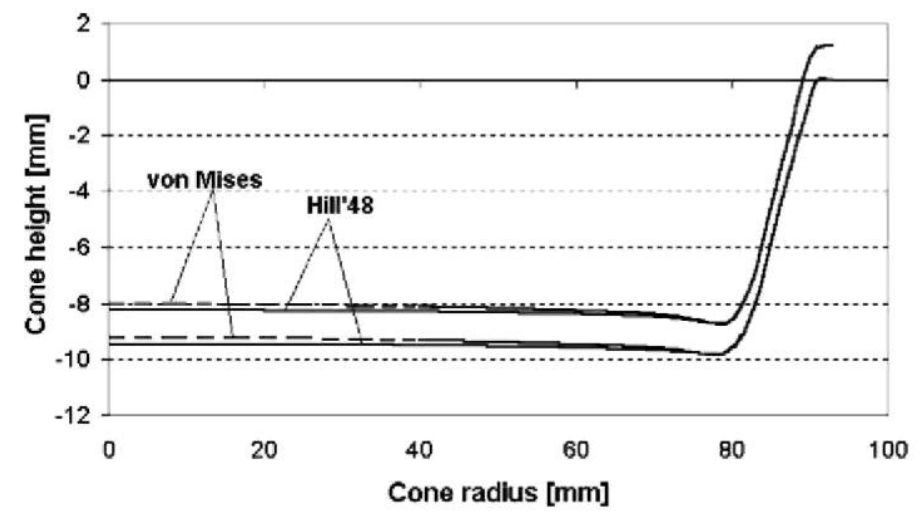

Fig. 25. Plastic strain mode during SPIF process.

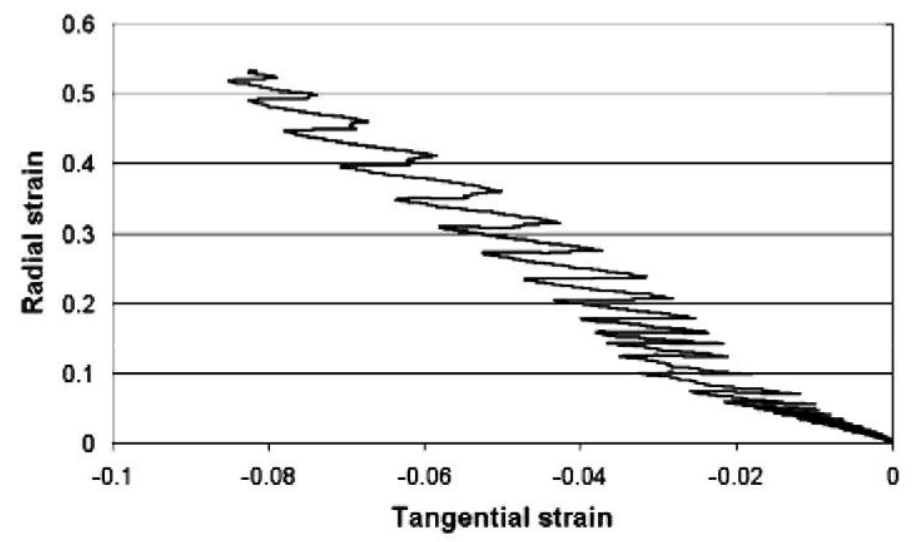

In the plane strain regions of the yield locus in both rolling or transversal directions, differences between all the surfaces clearly appear. Although this fact may explain the small geometrical differences predicted by the Hill and the Von Mises models concerning the cone bottom geometry, further research is needed. As Fig. 25 shows cyclic loading, it was important to check if the AA3003-0 material exhibits kinematic hardening behavior. The bi-axial equipment (Flores et al., 2005a,b) was used to perform reverse simple shear tests. The results plotted in Fig. 27 confirm that isotropic and kinematic hardening happen and that the chosen hardening model should be able to simulate variable hardening stagnation according the amount of pre-shear.

SPIF simulations have been performed with the Von Mises law coupled either with isotropic (Swift model) or mixed hardening law (isotropic Swift and kinematic Ziegler models) as the standard implicit Abaqus code used proposes neither Armstrong Frederick nor Teodosiu and Hu models. The Swift + Ziegler hardening law is described by Eqs. (12) and (13), the evolution of the back-stress $\underline{X}$ is defined by:

$\stackrel{\nabla}{X}=C_{A} \frac{1}{\bar{\sigma}}(\underline{\sigma}-\underline{X}) \cdot \dot{\bar{\varepsilon}}^{\mathrm{p}}-G_{A} \cdot \underline{X} \cdot \dot{\bar{\varepsilon}}^{\mathrm{p}}$ 
Fig. 26. Initial scaled yield locus of AA3003-O.

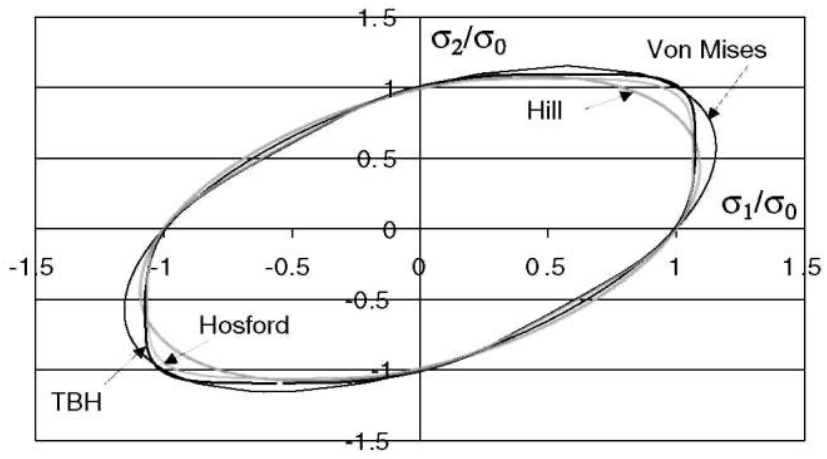

Fig. 27. Experimental Baushinger tests with $10 \%$ and $30 \%$ of pre-shear.

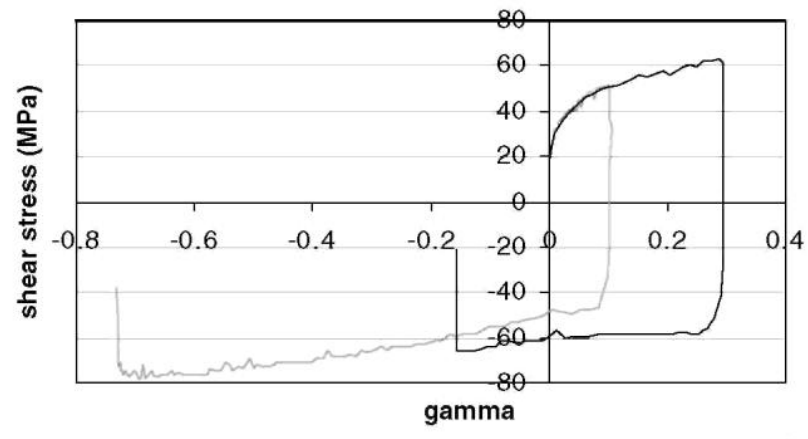

Baushinger 10\% - Baushinger 30\%

where $C_{A}$ is the initial kinematic hardening modulus, $G_{A}$ the rate at which the kinematic hardening modulus decreases with increasing plastic strain and $\bar{\sigma}$ the size of the yield surface which is a function of the equivalent plastic strain. As this model is not able to simulate the stagnation of the hardening in a reverse simple shear, the Teodosiu-Hu model coupled with Hill (1948) yield surface was also identified. Finally, Table 10 provides all the identified material parameters for the different models used in FE simulations.

Fig. 28 compares the prediction of mixed hardening models for a Bauschinger test in the RD. The "Hill + Teodosiu and $\mathrm{Hu}$ " curve is closer to experimental results than the "Von Mises + Ziegler" model.

During the Abaqus SPIF simulations, due to the fact that the mesh is rather coarse, especially in the circumferential direction, the contact force drops to zero within one contour when the tool is out of contact with the integration points of the elements. This does not happen in the real process where the tool always has contact with the sheet. To get rid of the unreasonable zero reaction force, a strategy has been adopted to calculate the average value of the force within one contour. Fig. 29 illustrates the average computed tool force for 80 contours provided by the two FE analyses, one relying on the Von Mises + S-wift model and the other one on the Von Mises + Swift + Ziegler model (see Table 9). Apparently, the reaction force increases for the first 10-12 contours, decreases a little until 20 contours and then remains rather stable for the rest of the process. Compared to experimental values, the FE results based on isotropic hardening are too high and the ones based on kinematic hardening are closer.

The FEM package Lagamine with Teodosiu and Hu model has been used by ULg team to compute a simplified approach. The strain history of Fig. 25 is applied on one finite element. To link the results of such a simulation to the force measured during the SPIF process is difficult. The accumulated plastic work during each tool path arc or "step" in the one finite element simulation is evaluated. As the average displacement applied on the element during one arc is known, a force representative of this arc can be computed as the average plastic work divided by the average displacement, as shown in Fig. 30. This computed force is maximum at the 30th arc because during this arc, the tool is in contact with the element. These "one finite element" simulations allow measuring the effect of the constitutive models. Two different laws are compared: the Von Mises + Swift + Ziegler model and the Hill + Teodosiu and Hu model. Clearly the first one predicts higher forces than the second one. So, used in whole FEM SPIF simulations, the Hill + Teodosiu model should decrease the predicted force and perhaps accurately predict the experimental results. However the results on such simplified approach are not quantitative. 
So the FEM SPIF simulations must confirm the accuracy of this last model. The only conclusion is the nonnegligible effect of the chosen mixed hardening model.

Table 10. Material parameters for AA3003-O for three different models

Von Mises + Isotropic Swift hardening

\begin{tabular}{|c|c|c|c|c|c|c|c|c|c|c|c|c|}
\hline \multicolumn{4}{|c|}{$K(\mathrm{MPa})$} & \multicolumn{4}{|l|}{$\varepsilon_{\mathrm{o}}$} & \multicolumn{4}{|l|}{$n$} & $m$ \\
\hline \multicolumn{4}{|c|}{180.0} & \multicolumn{4}{|l|}{0.00109} & \multicolumn{4}{|l|}{0.21} & 1 \\
\hline \multicolumn{13}{|c|}{ Von Mises + mixed hardening (Swift + Ziegler) } \\
\hline \multicolumn{3}{|c|}{$K(\mathrm{MPa})$} & \multicolumn{2}{|c|}{$\varepsilon_{\mathrm{o}}$} & \multicolumn{3}{|l|}{$n$} & $m$ & \multicolumn{2}{|l|}{$C_{A}$} & \multicolumn{2}{|l|}{$\boldsymbol{G}_{\boldsymbol{A}}$} \\
\hline \multicolumn{3}{|c|}{146.7} & \multicolumn{2}{|c|}{0.001496} & \multicolumn{3}{|l|}{0.229} & 1 & \multicolumn{2}{|l|}{240.6} & \multicolumn{2}{|c|}{11.2} \\
\hline \multicolumn{13}{|c|}{ Hill (1948) material parameters } \\
\hline \multicolumn{4}{|l|}{$\boldsymbol{F}$} & \multicolumn{4}{|l|}{$G$} & \multicolumn{3}{|l|}{$\boldsymbol{H}$} & \multicolumn{2}{|l|}{$N$} \\
\hline \multicolumn{4}{|c|}{1.224} & \multicolumn{4}{|l|}{1.194} & \multicolumn{3}{|c|}{0.806} & \multicolumn{2}{|c|}{3.92} \\
\hline \multicolumn{13}{|c|}{ Hill (1948) + mixed hardening (Teodosiu-Hu) } \\
\hline$R_{0}$ & $C_{R}$ & $R_{\mathrm{SAT}}$ & $C_{x}$ & $X_{\text {SAT0 }}$ & $m$ & $q$ & $C_{\mathrm{SD}}$ & $C_{\mathrm{sL}}$ & $S_{\mathrm{SAT}}$ & $n_{p}$ & $n_{\mathrm{L}}$ & $c_{\mathrm{p}}$ \\
\hline 43 & 73.3 & 25.22 & 300.2 & 8.45 & 0.399 & 1 & 6.94 & 0 & 70.67 & 130.92 & 1 & 0.97 \\
\hline
\end{tabular}

Fig. 28. Experimental results ad numerical predictions for a Bauschinger test with 10\% of pre-shear.

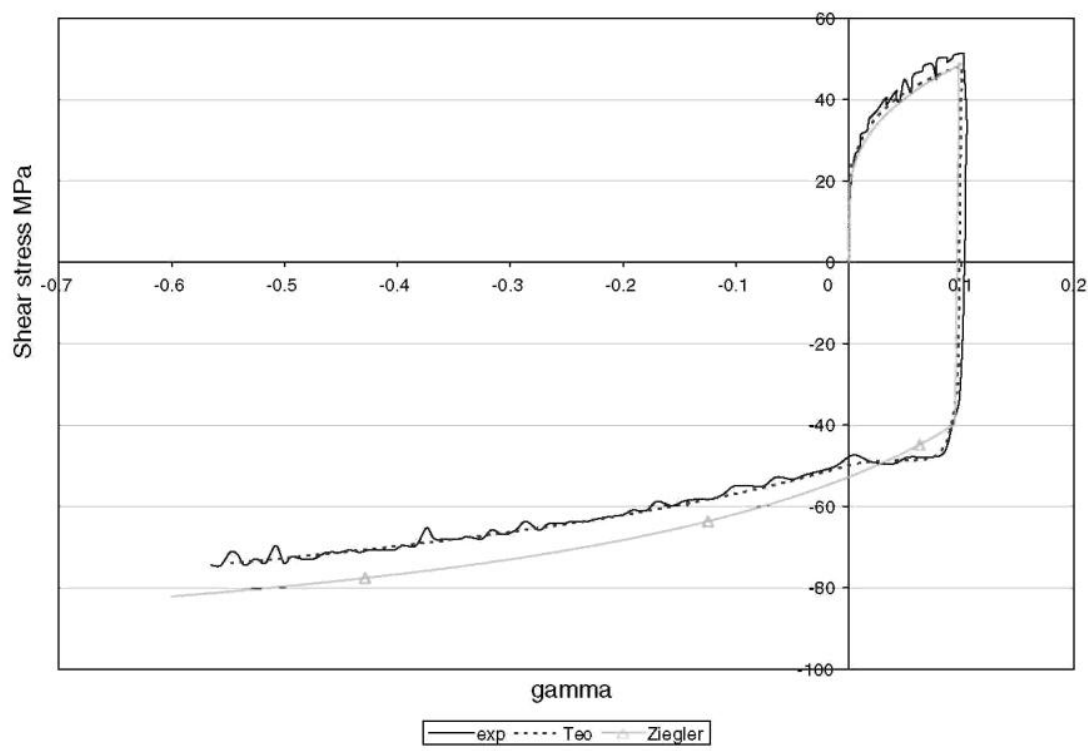

Fig. 29. Force measurements during SPIF process and Abaqus predictions.

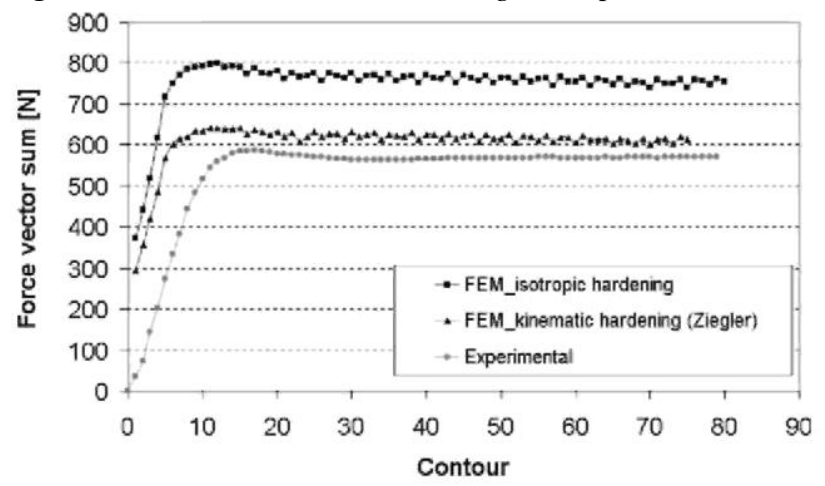


Fig. 30. Evolution of the representative tool force during each arc.

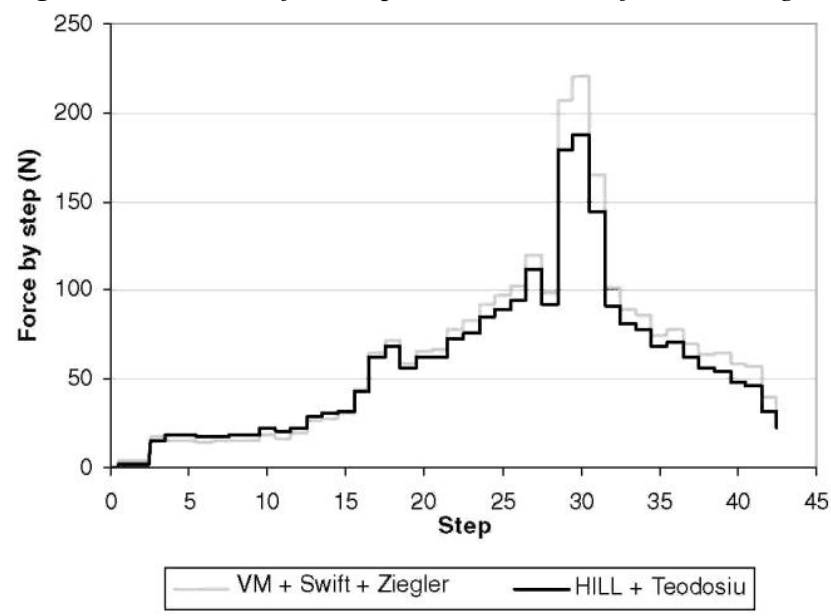

For the SPIF process, it is very important to anticipate the force acting on the tool, because the facility used is a conventional CNC milling machine, which is not dedicated to this process. The accurate prediction of the force is crucial to avoid any possible damage to the machine.

\section{Conclusions}

This paper underlines that the identification method of models is far from being trivial and without consequence for the accuracy of FEM model predictions. The well-known approach of using the Lankford coefficients to identify Hill parameters has been shown to provide quite rough material parameters and consequently poor FEM results. Another point is the fact that in the adjustment of the constitutive law parameters, one cannot decouple the yield locus shape from the hardening model, even if it would be easier for automatic material parameters identification.

When different choices of constitutive models are possible, the user must guide the research of material parameters knowing which models are available in his FEM code, the quality and the number of his experimental results and also his goal. Is it a first coarse analysis or does it require very accurate geometry or force predictions?

Two applications have been investigated. For plane strain test, the FEM simulation provided by the Hill, 1948 yield criterion coupled with the Teodosiu hardening model recovers experimental observation. The presence of horns at the free edges in the strain field is confirmed and the discrepancy between the experimental and numerical force-displacement curves can be explained.

The predicted force computed during a SPIF process according to the assumption of isotropic or kinematic hardening model has been presented. In this case, the mixed hardening model is clearly required to improve the numerical prediction of forces. Further simulations of the total process should validate which model better represents the actual material behavior.

\section{Acknowledgements}

As Research Director of the Fund for Scientific Research (Belgium), A.M. Habraken thanks this Belgian research fund for its support. The authors also thank the Belgian Federal Science Policy Office (Contract P5/08), the Institute for the Promotion of Innovation by Science and Technology in Flanders (IWT), the Walloon Region and Arcelor industry. 
Published in: International Journal of Plasticity (2007), vol. 23, iss. 3; pp. 420-449

Status: Postprint (Author's version)

\section{References}

Armstrong, P.J., Frederick, C.O., 1966. A mathematical representation of the multiaxial Bauschinger effect. GEGB Report RD/B/N731, Berkeley Nuclear Laboratories.

Banabic, B., Bunge, H.-J., PShlandt, K., Tekkaya, A.E., 2000. Formability of Metallic Materials. Springer, pp. 139-140.

Bouvier, S., Teodosiu, C., Haddadi, H., Tabacaru, V., 2003. Anisotropic work-hardening behaviour of structural steels and aluminium alloys at large strains. Journal de Physique IV 105, 215-222.

Bouvier, S., Haddadi, H., Levée, P., Teodosiu, C., 2005. Simple shear test: experimental techniques and characterizations of the plastic anisotropy of rolled sheets at large strains. Journal of Material Processing Technology 172 (1), 96-103.

Brunet, M., Morestin, F., Godereaux, S., 2001. Nonlinear kinematic hardening identification for anisotropic sheet metals with bendingunbending tests. Journal of Engineering Materials and Technology 123, 378-383.

Duchêne, L., de Montleau, P., El Houdaigui, F., Bouvier, S., Habraken, A.M., 2005. Analysis of texture evolution and hardening behavior during deep drawing with an improved mixed type FEM element. In: Smith, L.M., Pourboghrat, F., Yoon, J.-W., Stoughton, T.B. (Eds.) Proc. of the 6th Inter. Conf. and Workshop on Numerical Simulation of 3D Sheet Metal Forming Processes, On the Cutting Edge of Technology NUMISHEET, Detroit, MI, USA. AIP 778, 409-414.

Duflou, J.R., Szekeres, A., Vanherck, P., 2005. Force measurements for single point incremental forming: an experimental study. Advanced Materials Research 6-8, 441-448.

Flores, P., 2006, Development of experimental equipment and identification procedures for sheet metal constitutive laws. Ph.D. thesis, ULG, Liège.

Flores, P., Rondia, E., Habraken, A.M., 2005a. Development of an experimental equipment for the identification of constitutive laws (Special Issue). International Journal of Forming Processes 117-137.

Flores, P., Moureaux, P., Habraken, A.M., 2005b. Material identification using a bi-axial test machine. Advances in Experimental Mechanics IV 3-4, 91-97.

Fratini, L., Ambrogio, G., Di Lorenzo, R., Filice, L., Micari, F., 2004. Influence of mechanical properties of the sheet material on formability in single point incremental forming. CIRP Annals 53 (1), 207-210.

Habraken, A.M., Cescotto, S., 1990. An automatic remeshing technique for finite element simulation of forming processes. International Journal Numerical Methods in Engineering (30/8), 1503-1525.

Habraken, A.M., Duchêne, L., 2004. Anisotropic elasto-plastic finite element analysis using a stress-strain interpolation method based on a polycrystalline model. International Journal of Plasticity 20, 1525-1560.

Haddadi, H., Bouvier, S., Banu, M., Maier, C., Teodosiu, C., 2006. Towards an accurate description of the anisotropic behaviour of sheet metals under large plastic deformations: modelling, numerical analysis and identification. International Journal of Plasticity 22 (12), 22262271.

He, S., Van Bael, A., Van Houtte, P., Szekeres, A., Duflou, J.R., Henrard, C., Habraken, A.M., 2005a. Finite element modeling of incremental forming of aluminium sheets. Advanced Materials Research 6-8, 525-532.

He, S., Van Bael, A., Van Houtte, P., Tunckol, Y., Duflou, J., Henrard, C., Bouffioux, C., Habraken, A.M., 2005b. Effect of FEM choices in the modelling of incremental forming of aluminium sheets. In: Banabic, D. (Ed.), Proceedings of the 8th ESAFORM Conference on Material Forming. The Publishing House of the Romanian Academy, pp. 711-714.

Henrard, C., Habraken, A.M., Szekeres, A., Duflou, J.R., He, S., Van Bael, A., Van Houtte, P., 2005. Comparison of FEM simulations for the incremental forming process. Advanced Materials Research 6-8, 533-542.

Hill, R., 1948. A theory of the yielding and plastic flow of anisotropic materials. Proceedings of the Royal Society of London A 193, 281297.

Hosford, W.F., 1979. On yield loci of anisotropic cubic metals. In: Proceedings of the 7th North American Metalworking Conf. (NMRC), SME, Dearborn, MI, pp. 191-197.

Hosford, W.F., 1998. Reflections on the Dependence of Plastic Anisotropy on Texture. Materials Science and Engineering A 257, 1-8

$\mathrm{Hu}, \mathrm{W} ., 2005$. An orthotropic yield criterion in a 3-D general stress state. International Journal of Plasticity 21, 1771-1796. Iseki, H., 2001 An approximate deformation analysis and FEM analysis for the incremental bulging of sheet metal using a spherical roller. Journal of Materials Processing Technology 111, 150-154.

Jeswiet, J., Micari, F., Hirt, G., Bramley, A., Duflou, J., Allwood, J., 2005. Asymmetric single point incremental forming of sheet metal 
Published in: International Journal of Plasticity (2007), vol. 23, iss. 3; pp. 420-449

Status: Postprint (Author's version)

CIRP Annals, 54/2.

Kuwabara, T., Ikeda, S., Kuroda, K., 1998. Measurement and analysis of differential work hardening in cold-rolled steel sheet under bi-axial tension. Journal of Material Processing Technology 80-81, 517-523.

Lelotte, T., Gerday, A.F., Flores, P., Bouvier, S., Van Houtte, P., Habraken, A.M., 2005. Axes rotation during simple shear test: measurement and predictions. In: VIII Int. Conf. on Comp. Plasticity (COMPLAS VIII), Barcelona, Spain, pp. 1027-1030.

Li, S., Hoferlin, E., Van Bael, A., Van Houtte, P., Teodosiu, C, 2003. Finite element modeling of plastic anisotropy induced by texture and strain-path change. International Journal of Plasticity 19, 647-674.

Marquardt, D.W., 1963. An algorithm for least-squares estimation of non-linear parameters. Journal of the Society for Industrial and Applied Mathematics 11, 431-440.

Munhoven, S., Habraken, A.M., 1995. Application of an anisotropic yield locus based on texture to a deep, simulation of materials processing: theory, methods and applications. In: Shen, Dawson (Eds.), (C) 1995

Balkema, Rotterdam, pp. 767-772. ISBN 905410553 4. Peeters, B., Hoferlin, E., Van Houtte, P., Aernoudt, E., 2001. Assessment of crystal plasticity based calculation of lattice spin of polycrystalline metals for FE implementation. International Journal of Plasticity $17,819-836$.

Stoughton, T.B., Yoon, J.W., 2006. Review of Drucker's postulate and the issue of plastic stability in metal forming. International Journal of Plasticity 22, 391-433.

Teodosiu, C., Hu, Z., 1995. Evolution of the intragranular microstructure at moderate and large strains: modelling and computational significance. In: Shen, Dawson (Eds.), Simulation of Material Processing: Theory, Methods and Applications, (C) 1995 Balkema, Rotterdam, pp. 173-182. ISBN 9054105534.

Teodosiu, C., Hu, Z., 1998. Microstructure in the continuum modelling of plastic anisotropy. In: Carstensen, J.V., Leffers, T., Lorentzen, T., Pedersen, O.B., Sorensen, B.F., Winther, G. (Eds.), Proceedings of the $19^{\text {th }}$ Riso International Symposium on Materials Science: Modelling of Structure and Mechanics of Materials from Microscale to Product. Riso National Laboratory, Roskilde, Denmark, pp. 149-168.

Tong, W., 2006. A plane stress anisotropic plastic flow theory for orthotropic sheet metals. International Journal of Plasticity 22, $497-535$

Van Houtte, P., 1988. A comprehensive mathematical formulation of an extended Taylor-Bishop-Hill model featuring relaxed constraints, the Renouard-Wintenberger theory and a strain rate sensitivity model. Textures and Microstructures 8-9, 313-350.

Van Houtte, P., 2002. ODFLAM Software. Part of the latest version of MTM-FHM Software System, Katholieke Universiteit Leuven.

Wang, J., Wagoner, R.H., 2004. A new hexahedral solid element for 3D FEM simulation of sheet metal forming. In: Proceedings of the 8th Numiform Conference, vol. 712 (1), pp. 2181-2186.

Yoon, J.W., Barlat, F., Dick, R.E., Karabin, M.E., 2006. Prediction of six or eight ears in a drawn cup based on a new anisotropic yield function. International Journal of Plasticity 22, 174-193. 